\title{
Approximations of Numerical Method for Neutral Stochastic Functional Differential Equations with Markovian Switching
}

\author{
Hua Yang ${ }^{1}$ and Feng Jiang ${ }^{2}$ \\ ${ }^{1}$ School of Mathematics and Computer Science, Wuhan Polytechnic University, Wuhan 430023, China \\ ${ }^{2}$ School of Statistics and Mathematics, Zhongnan University of Economics and Law, Wuhan 430073, China \\ Correspondence should be addressed to Feng Jiang, fjiang78@gmail.com
}

Received 9 April 2012; Accepted 17 September 2012

Academic Editor: Zhenyu Huang

Copyright ( $) 2012$ H. Yang and F. Jiang. This is an open access article distributed under the Creative Commons Attribution License, which permits unrestricted use, distribution, and reproduction in any medium, provided the original work is properly cited.

Stochastic systems with Markovian switching have been used in a variety of application areas, including biology, epidemiology, mechanics, economics, and finance. In this paper, we study the Euler-Maruyama (EM) method for neutral stochastic functional differential equations with Markovian switching. The main aim is to show that the numerical solutions will converge to the true solutions. Moreover, we obtain the convergence order of the approximate solutions.

\section{Introduction}

Stochastic systems with Markovian switching have been successfully used in a variety of application areas, including biology, epidemiology, mechanics, economics, and finance [1]. As well as deterministic neutral functional differential equations and stochastic functional differential equations (SFDEs), most neutral stochastic functional differential equations with Markovian switching (NSFDEsMS) cannot be solved explicitly, so numerical methods become one of the powerful techniques. A number of papers have studied the numerical analysis of the deterministic neutral functional differential equations, for example, $[2,3]$ and references therein. The numerical solutions of SFDEs and stochastic systems with Markovian switching have also been studied extensively by many authors. Here we mention some of them, for example, [4-16]. moreover, Many well-known theorems in SFDEs are successfully extended to NSFDEs, for example, [17-24] discussed the stability analysis of the true solutions.

However, to the best of our knowledge, little is yet known about the numerical solutions for NSFDEsMS. In this paper we will extend the method developed by $[5,16]$ to 
NSFDEsMS and study strong convergence for the Euler-Maruyama approximations under the local Lipschitz condition, the linear growth condition, and contractive mapping. The three conditions are standard for the existence and uniqueness of the true solutions. Although the method of analysis borrows from [5], the existence of the neutral term and Markovian switching essentially complicates the problem. We develop several new techniques to cope with the difficulties which have risen from the two terms. Moreover, we also generalize the results in [19].

In Section 2, we describe some preliminaries and define the EM method for NSFDEsMS and state our main result that the approximate solutions strongly converge to the true solutions. The proof of the result is rather technical so we present several lemmas in Section 3 and then complete the proof in Section 4. In Section 5, under the global Lipschitz condition, we reveal the order of the convergence of the approximate solutions. Finally, a conclusion is made in Section 6.

\section{Preliminaries and EM Scheme}

Throughout this paper let $(\Omega, \mathscr{F}, \mathbb{P})$ be a complete probability space with a filtration $\left\{\mathscr{F}_{t}\right\}_{t \geq 0}$ satisfying the usual conditions, that is, it is right continuous and increasing while $\mathcal{F}_{0}$ contains all P-null sets. Let $w(t)=\left(w_{1}(t), \ldots, w_{m}(t)\right)^{T}$ be an $m$-dimensional Brownian motion defined on the probability space. Let $|\cdot|$ be the Euclidean norm in $\mathbb{R}^{n}$. Let $\mathbb{R}_{+}=[0, \infty)$, and let $\tau>0$. Denoted by $C\left([-\tau, 0], \mathbb{R}^{n}\right)$ the family of continuous functions from $[-\tau, 0]$ to $\mathbb{R}^{n}$ with the norm $\|\varphi\|=\sup _{-\tau \leq \theta \leq 0}|\varphi(\theta)|$. Let $p>0$ and $L_{\mathscr{f}_{0}}^{p}\left([-\tau, 0] ; \mathbb{R}^{n}\right)$ be the family of $\mathcal{F}_{0^{-}}$ measurable $C\left([-\tau, 0] ; \mathbb{R}^{n}\right)$-valued random variables $\xi$ such that $\mathbb{E}\|\xi\|^{p}<\infty$. If $x(t)$ is an $\mathbb{R}^{n_{-}}$ valued stochastic process on $t \in[-\tau, \infty)$, we let $x_{t}=\{x(t+\theta):-\tau \leq \theta \leq 0\}$ for $t \geq 0$.

Let $r(t), t \geq 0$, be a right-continuous Markov chain on the probability space taking values in a finite state space $S=\{1,2, \ldots, N\}$ with the generator $\Gamma=\left(\gamma_{i j}\right)_{N \times N}$ given by

$$
P\{r(t+\Delta)=j \mid r(t)=i\}= \begin{cases}\gamma_{i j} \Delta+o(\Delta) & \text { if } i \neq j, \\ 1+\gamma_{i j} \Delta+o(\Delta) & \text { if } i=j\end{cases}
$$

where $\Delta>0$. Here $\gamma_{i j} \geq 0$ is the transition rate from $i$ to $j$ if $i \neq j$ while $\gamma_{i i}=-\sum_{i \neq j} \gamma_{i j}$. We assumethat the Markov chain $r(\cdot)$ is independent of the Brownian motion $w(\cdot)$. It is well known that almost every sample path of $r(\cdot)$ is a right-continuous step function with finite number of simple jumps in any finite subinterval of $\mathfrak{R}_{+}=[0, \infty)$.

In this paper, we consider the $n$-dimensional NSFDEsMS

$$
d\left[x(t)-u\left(x_{t}, r(t)\right)\right]=f\left(x_{t}, r(t)\right) d t+g\left(x_{t}, r(t)\right) d w(t), \quad t \geq 0,
$$

with initial data $x_{0}=\xi \in L_{\mathscr{q}_{0}}^{p}\left([-\tau, 0] ; \mathbb{R}^{n}\right)$ and $r(0)=i_{0} \in S$, where $f: C\left([-\tau, 0] ; \mathbb{R}^{n}\right) \rightarrow \mathbb{R}^{n}$, $g: C\left([-\tau, 0] ; \mathbb{R}^{n}\right) \rightarrow \mathbb{R}^{n \times m}$ and $u: C\left([-\tau, 0] ; \mathbb{R}^{n}\right) \rightarrow \mathbb{R}^{n}$. As a standing hypothesis we assume that both $f$ and $g$ are sufficiently smooth so that (2.2) has a unique solution. We refer the reader to Mao $[10,12]$ for the conditions on the existence and uniqueness of the solution $x(t)$. The initial data $\xi$ and $i_{0}$ could be random, but the Markov property ensures that it is sufficient to consider only the case when both $x_{0}$ and $i_{0}$ are constants.

To analyze the Euler-Maruyama (EM) method, we need the following lemma (see $[6,7,10-12,16])$. 
Lemma 2.1. Given $\Delta>0$, let $r_{k}^{\Delta}=r(k \Delta)$ for $k \geq 0$. Then $\left\{r_{k}^{\Delta}, k=0,1,2, \ldots\right\}$ is a discrete Markov chain with the one-step transition probability matrix

$$
P(\Delta)=\left(P_{i j}(\Delta)\right)_{N \times N}=e^{\Delta \Gamma} .
$$

For the completeness, we give the simulation of the Markov chain as follows. Given a stepsize $\Delta>0$, we compute the one-step transition probability matrix

$$
P(\Delta)=\left(P_{i j}(\Delta)\right)_{N \times N}=e^{\Delta \Gamma} .
$$

Let $r_{0}^{\Delta}=i_{0}$ and generate a random number $\xi_{1}$ which is uniformly distributed in $[0,1]$. Define

$$
r_{1}^{\Delta}= \begin{cases}i_{1}, & \text { if } i_{1} \in S-\{N\} \text { such that } \sum_{j=1}^{i_{1}-1} P_{i_{0}, j}(\Delta) \leq \xi_{1}<\sum_{j=1}^{i_{1}} P_{i_{0}, j}(\Delta), \\ N, & \text { if } \sum_{j=1}^{N-1} P_{i_{0}, j}(\Delta) \leq \xi_{1},\end{cases}
$$

where we set $\sum_{i=1}^{0} P_{i_{0}, j}(\Delta)=0$ as usual. Generate independently a new random number $\xi_{2}$ which is again uniformly distributed in $[0,1]$, and then define

$$
r_{2}^{\Delta}= \begin{cases}i_{2}, & \text { if } i_{2} \in S-\{N\} \text { such that } \sum_{j=1}^{i_{2}-1} P_{r_{1}^{\Delta}, j}(\Delta) \leq \xi_{2}<\sum_{j=1}^{i_{2}} P_{r_{1}^{\Delta}, j}(\Delta), \\ N, & \text { if } \sum_{j=1}^{N-1} P_{r_{1}^{\Delta}, j}(\Delta) \leq \xi_{2} .\end{cases}
$$

Repeating this procedure a trajectory of $\left\{r_{k}^{\Delta}, k=0,1,2, \ldots\right\}$ can be generated. This procedure can be carried out independently to obtain more trajectories.

Now we can define the Euler-Maruyama (EM) approximate solution for (2.2) on the finite time interval $[0, T]$. Without loss of any generality, we may assume that $T / \tau$ is a rational number; otherwise we may replace $T$ by a larger number. Let the step size $\Delta \in(0,1)$ be a fraction of $\tau$ and $T$, namely, $\Delta=\tau / N=T / M$ for some integers $N>\tau$ and $M>T$. The explicit discrete EM approximate solution $\bar{y}(k \Delta), k \geq-N$ is defined as follows:

$$
\begin{gathered}
\bar{y}(k \Delta)=\xi(k \Delta), \quad-N \leq k \leq 0, \\
\bar{y}((k+1) \Delta)=\bar{y}(k \Delta)+u\left(\bar{y}_{k \Delta^{\prime}} r_{k}^{\Delta}\right)-u\left(\bar{y}_{(k-1) \Delta^{\prime}} r_{k-1}^{\Delta}\right)+f\left(\bar{y}_{k \Delta^{\prime}} r_{k}^{\Delta}\right) \Delta \\
+g\left(\bar{y}_{k \Delta}, r_{k}^{\Delta}\right) \Delta w_{k}, \quad 0 \leq k \leq M-1,
\end{gathered}
$$


where $\Delta w_{k}=w((k+1) \Delta)-w(k \Delta)$ and $\bar{y}_{k \Delta}=\left\{\bar{y}_{k \Delta}(\theta):-\tau \leq \theta \leq 0\right\}$ is a $C\left([-\tau, 0] ; \mathbb{R}^{n}\right)$-valued random variable defined by

$$
\begin{aligned}
\bar{y}_{k \Delta}(\theta) & =\bar{y}((k+i) \Delta)+\frac{\theta-i \Delta}{\Delta}[\bar{y}((k+i+1) \Delta)-\bar{y}((k+i) \Delta)] \\
& =\frac{\Delta-(\theta-i \Delta)}{\Delta} \bar{y}((k+i) \Delta)+\frac{\theta-i \Delta}{\Delta} \bar{y}((k+i+1) \Delta),
\end{aligned}
$$

for $i \Delta \leq \theta \leq(i+1) \Delta, i=-N,-N+1, \ldots,-1$, where in order for $\bar{y}_{-\Delta}$ to be well defined, we set $\bar{y}(-(N+1) \Delta)=\xi(-N \Delta)$.

That is, $\bar{y}_{k \Delta}(\cdot)$ is the linear interpolation of $\bar{y}((k-N) \Delta), \bar{y}((k-N+1) \Delta), \ldots, \bar{y}(k \Delta)$. We hence have

$$
\begin{aligned}
\left|\bar{y}_{k \Delta}(\theta)\right| & =\frac{\Delta-(\theta-i \Delta)}{\Delta}|\bar{y}((k+i) \Delta)|+\frac{\theta-i \Delta}{\Delta}|\bar{y}((k+i+1) \Delta)| \\
& \leq|\bar{y}((k+i) \Delta)| \vee|\bar{y}((k+i+1) \Delta)| .
\end{aligned}
$$

We therefore obtain

$$
\left|\bar{y}_{k \Delta}(\theta)\right|=\max _{-N \leq i \leq 0}|\bar{y}((k+i) \Delta)|, \quad \text { for any } k=-1,0,1, \ldots, M-1
$$

It is obvious that $\left\|\bar{y}_{-\Delta}\right\| \leq\left\|\bar{y}_{0}\right\|$.

In our analysis it will be more convenient to use continuous-time approximations. We hence introduce the $C\left([-\tau, 0] ; \mathbb{R}^{n}\right)$-valued step process

$$
\begin{gathered}
\bar{y}_{t}=\sum_{k=0}^{M-2} \bar{y}_{k \Delta} 1_{[k \Delta,(k+1) \Delta)}(t)+\bar{y}_{(M-1) \Delta} 1_{[(M-1) \Delta, M \Delta]}(t), \\
\bar{r}(t)=\sum_{k=0}^{M-1} r_{k}^{\Delta} 1_{[k \Delta,(k+1) \Delta)}(t),
\end{gathered}
$$

and we define the continuous EM approximate solution as follows: let $y(t)=\xi(t)$ for $-\tau \leq t \leq$ 0 , while for $t \in[k \Delta,(k+1) \Delta], k=0,1, \ldots, M-1$,

$$
\begin{aligned}
y(t)= & \xi(0)+u\left(\bar{y}_{(k-1) \Delta}+\frac{t-k \Delta}{\Delta}\left(\bar{y}_{k \Delta}-\bar{y}_{(k-1) \Delta}\right), r_{k}^{\Delta}\right)-u\left(\bar{y}_{-\Delta}, r_{0}^{\Delta}\right) \\
& +\int_{0}^{t} f\left(\bar{y}_{s}, \bar{r}(s)\right) d s+\int_{0}^{t} g\left(\bar{y}_{s}, \bar{r}(s)\right) d w(s) .
\end{aligned}
$$


Clearly, (2.12) can also be written as

$$
\begin{aligned}
y(t)= & \bar{y}(k \Delta)+u\left(\bar{y}_{(k-1) \Delta}+\frac{t-k \Delta}{\Delta}\left(\bar{y}_{k \Delta}-\bar{y}_{(k-1) \Delta}\right), r_{k}^{\Delta}\right)-u\left(\bar{y}_{(k-1) \Delta}, r_{k-1}^{\Delta}\right) \\
& +\int_{k \Delta}^{t} f\left(\bar{y}_{s}, \bar{r}(s)\right) d s+\int_{k \Delta}^{t} g\left(\bar{y}_{s}, \bar{r}(s)\right) d w(s) .
\end{aligned}
$$

In particular, this shows that $y(k \Delta)=\bar{y}(k \Delta)$, that is, the discrete and continuous EM approximate solutions coincide at the grid points. We know that $y(t)$ is not computable because it requires knowledge of the entire Brownian path, not just its $\Delta$-increments. However, $y(k \Delta)=\bar{y}(k \Delta)$, so the error bound for $y(t)$ will automatically imply the error bound for $\bar{y}(k \Delta)$. It is then obvious that

$$
\left\|\bar{y}_{k \Delta}\right\| \leq\left\|y_{k \Delta}\right\|, \quad \forall k=0,1,2, \ldots, M-1 .
$$

Moreover, for any $t \in[0, T]$,

$$
\begin{aligned}
\sup _{0 \leq t \leq T}\left\|\bar{y}_{t}\right\| & =\sup _{0 \leq k \leq M-1}\left\|\bar{y}_{k \Delta}\right\| \leq \sup _{0 \leq k \leq M-1}\left\|y_{k \Delta}\right\| \\
& =\sup _{0 \leq k \leq M-1-\tau \leq \theta \leq 0} \sup _{0}|y(k \Delta+\theta)| \\
& \leq \sup _{0 \leq t \leq T-\tau \leq \theta \leq 0} \sup _{0}|y(t+\theta)| \\
& \leq \sup _{-\tau \leq s \leq T}|y(s)|,
\end{aligned}
$$

and letting $[t / \Delta]$ be the integer part of $t / \Delta$, then

$$
\left\|\bar{y}_{t}\right\|=\left\|\bar{y}_{[t / \Delta] \Delta}\right\| \leq\left\|y_{[t / \Delta] \Delta}\right\| \leq \sup _{-\tau \leq s \leq t}|y(s)| .
$$

These properties will be used frequently in what follows, without further explanation.

For the existence and uniqueness of the solution of (2.2) and the boundedness of the solution's moments, we impose the following hypotheses (e.g., see [11]).

Assumption 2.2. For each integer $j \geq 1$ and $i \in S$, there exists a positive constant $C_{j}$ such that

$$
\left|f(\varphi, i)-f(\psi, i)^{2}\right| \vee\left|g(\varphi, i)-g(\psi, i)^{2}\right| \leq C_{j}\|\varphi-\psi\|^{2}
$$

for $\varphi, \psi \in C\left([-\tau, 0] ; \mathbb{R}^{n}\right)$ with $\|\varphi\| \vee\|\psi\| \leq j$.

Assumption 2.3. There is a constant $K>0$ such that

$$
|f(\varphi, i)|^{2} \vee|g(\varphi, i)|^{2} \leq K\left(1+\|\varphi\|^{2}\right),
$$

for $\varphi \in C\left([-\tau, 0] ; \mathbb{R}^{n}\right)$ and $i \in S$. 
Assumption 2.4. There exists a constant $\kappa \in(0,1)$ such that for all $\varphi, \psi \in C\left([-\tau, 0] ; \mathbb{R}^{n}\right)$ and $i \in S$,

$$
|u(\varphi, i)-u(\psi, i)| \leq \kappa\|\varphi-\psi\|,
$$

for $\varphi, \psi \in C\left([-\tau, 0] ; \mathbb{R}^{n}\right)$ and $u(0, i)=0$.

We also impose the following condition on the initial data.

Assumption 2.5. $\xi \in L_{\mathscr{\mp}_{0}}^{p}\left([-\tau, 0] ; \mathbb{R}^{n}\right)$ for some $p \geq 2$, and there exists a nondecreasing function $\alpha(\cdot)$ such that

$$
\mathbb{E}\left(\sup _{-\tau \leq s \leq t \leq 0}|\xi(t)-\xi(s)|^{2}\right) \leq \alpha(t-s),
$$

with the property $\alpha(s) \rightarrow 0$ as $s \rightarrow 0$.

From Mao and Yuan [11], we may therefore state the following theorem.

Theorem 2.6. Let $p \geq 2$. If Assumptions 2.3-2.5 are satisfied, then

$$
\mathbb{E}\left(\sup _{-\tau \leq t \leq T}|x(t)|^{p}\right) \leq H_{\kappa, p, T, K, \xi '}
$$

for any $T>0$, where $H_{\kappa, p, T, K, \xi}$ is a constant dependent on $\mathcal{\kappa}, p, T, K, \xi$.

The primary aim of this paper is to establish the following strong mean square convergence theorem for the EM approximations.

Theorem 2.7. If Assumptions 2.2-2.5 hold,

$$
\lim _{\Delta \rightarrow 0} \mathbb{E}\left(\sup _{0 \leq t \leq T}|x(t)-y(t)|^{2}\right)=0 .
$$

The proof of this theorem is very technical, so we present some lemmas in the next section, and then complete the proof in the sequent section.

\section{Lemmas}

Lemma 3.1. If Assumptions 2.3-2.5 hold, for any $p \geq 2$, there exists a constant $H(p)$ such that

$$
\mathbb{E}\left(\sup _{-\tau \leq t \leq T}|y(t)|^{p}\right) \leq H(p),
$$

where $H(p)$ is independent of $\Delta$. 
Proof. For $t \in[k \Delta,(k+1) \Delta], k=0,1,2, \ldots, M-1$, set $\tilde{y}(t):=y(t)-u\left(\bar{y}_{(k-1) \Delta}+(t-k \Delta)\left(\bar{y}_{k \Delta}-\right.\right.$ $\left.\left.\bar{y}_{(k-1) \Delta}\right) / \Delta, r_{k}^{\Delta}\right)$ and

$$
h(t):=\mathbb{E}\left(\sup _{-\tau \leq s \leq t}|y(s)|^{p}\right), \quad \tilde{h}(t):=\mathbb{E}\left(\sup _{0 \leq s \leq t}|\tilde{y}(s)|^{p}\right) .
$$

Recall the inequality that for $p \geq 1$ and any $\varepsilon>0,|x+y|^{p} \leq(1+\varepsilon)^{p-1}\left(|x|^{p}+\varepsilon^{1-p}|y|^{p}\right)$. Then we have, from Assumption 2.4,

$$
\begin{aligned}
|y(t)|^{p} & \leq(1+\varepsilon)^{p-1}\left(|\tilde{y}(t)|^{p}+\varepsilon^{1-p}\left|u\left(\bar{y}_{(k-1) \Delta}+\frac{(t-k \Delta)\left(\bar{y}_{k \Delta}-\bar{y}_{(k-1) \Delta}\right)}{\Delta}, r_{k}^{\Delta}\right)\right|^{p}\right) \\
& \leq(1+\varepsilon)^{p-1}\left(|\tilde{y}(t)|^{p}+\varepsilon^{1-p} \mathcal{K}^{p}\left\|\bar{y}_{(k-1) \Delta}+\frac{t-k \Delta}{\Delta}\left(\bar{y}_{k \Delta}-\bar{y}_{(k-1) \Delta}\right)\right\|^{p}\right) .
\end{aligned}
$$

By $\left\|\bar{y}_{-\Delta}\right\| \leq\left\|\bar{y}_{0}\right\|$, noting $k=0,1,2, \ldots, M-1$,

$$
\begin{aligned}
\left\|\bar{y}_{(k-1) \Delta}+\frac{t-k \Delta}{\Delta}\left(\bar{y}_{k \Delta}-\bar{y}_{(k-1) \Delta}\right)\right\|^{p} & \leq\left|\frac{(k+1) \Delta-t}{\Delta}\left\|\bar{y}_{(k-1) \Delta}\right\|+\frac{t-k \Delta}{\Delta}\left\|\bar{y}_{k \Delta}\right\|\right|^{p} \\
& \leq\left[\frac{(k+1) \Delta-t}{\Delta}\left(\sup _{-\tau \leq s \leq t}|y(s)|\right)+\frac{t-k \Delta}{\Delta}\left(\sup _{-\tau \leq s \leq t}|y(s)|\right)\right]^{p} \\
& \leq \sup _{-\tau \leq s \leq t}|y(s)|^{p} .
\end{aligned}
$$

Consequently, choose $\varepsilon=\kappa /(1-\kappa)$, then

$$
|y(t)|^{p} \leq(1-\kappa)^{1-p}|\tilde{y}(t)|^{p}+\kappa\left(\sup _{-\tau \leq s \leq t}|y(s)|^{p}\right) .
$$

Hence,

$$
\begin{aligned}
h(t) & \leq \mathbb{E}\|\xi\|^{p}+\mathbb{E}\left(\sup _{0 \leq s \leq t}|y(s)|^{p}\right) \\
& \leq \mathbb{E}\|\xi\|^{p}+\kappa h(t)+(1-\kappa)^{1-p} \widetilde{h}(t),
\end{aligned}
$$

which implies

$$
h(t) \leq \frac{\mathbb{E}\|\xi\|^{p}}{1-\kappa}+\frac{\tilde{h}(t)}{(1-\kappa)^{p}}
$$


Since

$$
\tilde{y}(t)=\tilde{y}(0)+\int_{0}^{t} f\left(\bar{y}_{s}, \bar{r}(s)\right) d s+\int_{0}^{t} g\left(\bar{y}_{s}, \bar{r}(s)\right) d w(s),
$$

with $\tilde{y}(0)=\bar{y}(0)-u\left(\bar{y}_{-\Delta}\right)$, by the Hölder inequality, we have

$$
|\widetilde{y}(t)|^{p} \leq 3^{p-1}\left[|\tilde{y}(0)|^{p}+t^{p-1} \int_{0}^{t}\left|f\left(\bar{y}_{s}, \bar{r}(s)\right)\right|^{p} d s+\left|\int_{0}^{t} g\left(\bar{y}_{s}, \bar{r}(s)\right) d w(s)\right|^{p}\right] .
$$

Hence, for any $t_{1} \in[0, T]$,

$$
\tilde{h}\left(t_{1}\right) \leq 3^{p-1}\left[\mathbb{E}|\tilde{y}(0)|^{p}+T^{p-1} \mathbb{E} \int_{0}^{t_{1}}\left|f\left(\bar{y}_{s^{\prime}} \bar{r}(s)\right)\right|^{p} d s+\mathbb{E}\left(\sup _{0 \leq t \leq t_{1}}\left|\int_{0}^{t} g\left(\bar{y}_{s^{\prime}} \bar{r}(s)\right) d w(s)\right|^{p}\right)\right] .
$$

By Assumption 2.4 and the fact $\left\|\bar{y}_{-\Delta}\right\| \leq\left\|\bar{y}_{0}\right\|$, we compute that

$$
\begin{aligned}
\mathbb{E}|\widetilde{y}(0)|^{p} & =\mathbb{E}\left|\bar{y}(0)-u\left(\bar{y}_{-\Delta}, r_{0}^{\Delta}\right)\right|^{p} \\
& \leq \mathbb{E}\left(|\bar{y}(0)|+\kappa\left\|\bar{y}_{-\Delta}\right\|\right)^{p} \\
& \leq \mathbb{E}\left(|\bar{y}(0)|+\kappa\left\|\bar{y}_{0}\right\|\right)^{p} \\
& \leq \mathbb{E}(|\xi(0)|+\kappa\|\xi\|)^{p} \\
& \leq(1+\kappa)^{p} \mathbb{E}\|\xi\|^{p}
\end{aligned}
$$

Assumption 2.3 and the Hölder inequality give

$$
\begin{aligned}
\mathbb{E} \int_{0}^{t_{1}}\left|f\left(\bar{y}_{s}, \bar{r}(s)\right)\right|^{p} d s & \leq \mathbb{E} \int_{0}^{t_{1}} K^{p / 2}\left(1+\left\|\bar{y}_{s}\right\|^{2}\right)^{p / 2} d s \\
& \leq K^{p / 2} 2^{(p-2) / 2} \mathbb{E} \int_{0}^{t_{1}}\left(1+\left\|\bar{y}_{s}\right\|^{p}\right) d s \\
& \leq K^{p / 2} 2^{(p-2) / 2}\left[T+\int_{0}^{t_{1}} \mathbb{E}\left(\sup _{-\tau \leq t \leq s}|y(t)|^{p} d s\right)\right] .
\end{aligned}
$$


Applying the Burkholder-Davis-Gundy inequality, the Hölder inequality and Assumption 2.3 yield

$$
\begin{aligned}
\mathbb{E}\left(\sup _{0 \leq t \leq t_{1}}\left|\int_{0}^{t} g\left(\bar{y}_{s}, \bar{r}(s)\right) d w(s)\right|^{p}\right) & \leq C_{p} \mathbb{E}\left(\int_{0}^{t_{1}}\left|g\left(\bar{y}_{s^{\prime}} \bar{r}(s)\right)\right|^{2} d s\right)^{p / 2} \\
& \leq C_{p} T^{(p-2) / 2} \mathbb{E} \int_{0}^{t_{1}}\left|g\left(\bar{y}_{s}, \bar{r}(s)\right)\right|^{p} d s \\
& \leq C_{p} T^{(p-2) / 2} \mathbb{E} \int_{0}^{t_{1}} K^{p / 2}\left(1+\left\|\bar{y}_{s}\right\|^{2}\right)^{p / 2} d s \\
& \leq C_{p} T^{(p-2) / 2} K^{p / 2} 2^{(p-2) / 2} \mathbb{E} \int_{0}^{t_{1}}\left(1+\left\|\bar{y}_{s}\right\|^{p}\right) d s \\
& \leq C_{p} T^{(p-2) / 2} K^{p / 2} 2^{(p-2) / 2}\left[T+\int_{0}^{t_{1}} \mathbb{E}\left(\sup _{-\tau \leq t \leq s}|y(t)|^{p}\right) d s\right],
\end{aligned}
$$

where $C_{p}$ is a constant dependent only on $p$. Substituting (3.11), (3.12), and (3.13) into (3.10) gives

$$
\begin{aligned}
\tilde{h}\left(t_{1}\right) \leq & 3^{p-1}\left[(1+\kappa)^{p} \mathbb{E}\|\xi\|^{p}+K^{p / 2} 2^{(p-2) / 2} T^{p}+C_{p}(2 T)^{(p-2) / 2} K^{p / 2} T\right] \\
& +3^{p-1}\left[K^{p / 2} 2^{(p-2) / 2} T^{p-1}+C_{p}(2 T)^{(p-2) / 2} K^{p / 2}\right] \int_{0}^{t_{1}} \mathbb{E}\left(\sup _{-\tau \leq t \leq s}|y(t)|^{p}\right) d s \\
= & : C_{1}+C_{2} \int_{0}^{t_{1}} h(s) d s .
\end{aligned}
$$

Hence from (3.7), we have

$$
\begin{aligned}
h\left(t_{1}\right) & \leq \frac{\mathbb{E}\|\xi\|^{p}}{1-\kappa}+\frac{1}{(1-\kappa)^{p}}\left[C_{1}+C_{2} \int_{0}^{t_{1}} h(s) d s\right] \\
& \leq \frac{\mathbb{E}\|\xi\|^{p}}{1-\kappa}+\frac{C_{1}}{(1-\kappa)^{p}}+\frac{C_{2}}{(1-\kappa)^{p}} \int_{0}^{t_{1}} h(s) d s .
\end{aligned}
$$

By the Gronwall inequality we find that

$$
h(T) \leq\left[\frac{\mathbb{E}\|\xi\|^{p}}{1-\kappa}+\frac{C_{1}}{(1-\kappa)^{p}}\right] e^{C_{2} T /(1-\kappa)^{p}}
$$

From the expressions of $C_{1}$ and $C_{2}$, we know that they are positive constants dependent only on $\xi, \kappa, K, p$, and $T$, but independent of $\Delta$. The proof is complete. 
Lemma 3.2. If Assumptions 2.3-2.5 hold, then for any integer $l>1$,

$$
\mathbb{E}\left(\sup _{0 \leq k \leq M-1}\left\|\bar{y}_{k \Delta}-\bar{y}_{(k-1) \Delta}\right\|^{2}\right) \leq c_{1}^{\prime}+c_{1} \alpha(\Delta)+\bar{c}_{1}(l) \Delta^{(l-1) / l}=: \gamma(\Delta),
$$

where $c_{1}=1 /(1-2 \kappa), c_{1}^{\prime}=(8 \kappa /(1-2 \kappa)) H(2)$, and $\bar{c}_{1}(l)$ is a constant dependent on $l$ but independent of $\Delta$.

Proof. For $\theta \in[i \Delta,(i+1) \Delta]$, where $i=-N,-(N+1), \ldots,-1$, from (2.7),

$$
\begin{aligned}
\left|\bar{y}_{k \Delta}-\bar{y}_{(k-1) \Delta}\right| \leq & \frac{(i+1) \Delta-\theta}{\Delta}|\bar{y}((k+i) \Delta)-\bar{y}((k-1+i) \Delta)| \\
& +\frac{\theta-i \Delta}{\Delta}|\bar{y}((k+i+1) \Delta)-\bar{y}((k+i) \Delta)| \\
\leq & |\bar{y}((k+i) \Delta)-\bar{y}((k-1+i) \Delta)| \vee|\bar{y}((k+i+1) \Delta)-\bar{y}((k+i) \Delta)|,
\end{aligned}
$$

so

$$
\left\|\bar{y}_{k \Delta}-\bar{y}_{(k-1) \Delta}\right\| \leq \sup _{-N \leq i \leq 0}|\bar{y}((k+i) \Delta)-\bar{y}((k-1+i) \Delta)| .
$$

We therefore have

$$
\begin{aligned}
\mathbb{E}\left(\sup _{0 \leq k \leq M-1}\left\|\bar{y}_{k \Delta}-\bar{y}_{(k-1) \Delta}\right\|^{2}\right) & \leq \mathbb{E}\left[\sup _{0 \leq k \leq M-1}\left(\sup _{-N \leq i \leq 0}|\bar{y}((k+i) \Delta)-\bar{y}((k-1+i) \Delta)|^{2}\right)\right] \\
& \leq \mathbb{E}\left(\sup _{-N \leq k \leq M-1}|\bar{y}(k \Delta)-\bar{y}((k-1) \Delta)|^{2}\right)
\end{aligned}
$$

When $-N \leq k \leq 0$, by Assumption 2.5 and $\bar{y}(-(N+1) \Delta)=\xi(-N \Delta)$,

$$
\begin{aligned}
\mathbb{E}\left(\sup _{-N \leq k \leq 0}|\bar{y}(k \Delta)-\bar{y}((k-1) \Delta)|^{2}\right) & \leq \mathbb{E}\left(\sup _{-N \leq k \leq 0}|\xi(k \Delta)-\xi((k-1) \Delta)|^{2}\right) \\
& \leq \alpha(\Delta) .
\end{aligned}
$$


When $1 \leq k \leq M-1$, from (2.13), we have

$$
\begin{aligned}
\bar{y}(k \Delta)-\bar{y}((k-1) \Delta)= & u\left(\bar{y}_{(k-1) \Delta}, r_{k-1}^{\Delta}\right)-u\left(\bar{y}_{(k-2) \Delta}, r_{k-2}^{\Delta}\right)+f\left(\bar{y}_{(k-1) \Delta}, r_{k-1}^{\Delta}\right) \Delta \\
& +g\left(\bar{y}_{(k-1) \Delta}, r_{k-1}^{\Delta}\right) \Delta w_{k-1}
\end{aligned}
$$

Recall the elementary inequality, for any $x, y>0$ and $\varepsilon \in(0,1),(x+y)^{2} \leq x^{2} / \varepsilon+y^{2} /(1-\varepsilon)$. Then

$$
\begin{aligned}
\mid \bar{y}(k \Delta) & -\left.\bar{y}((k-1) \Delta)\right|^{2} \\
\leq & \frac{1}{\varepsilon}\left|u\left(\bar{y}_{(k-1) \Delta}, r_{k-1}^{\Delta}\right)-u\left(\bar{y}_{(k-2) \Delta}, r_{k-1}^{\Delta}\right)+u\left(\bar{y}_{(k-2) \Delta}, r_{k-1}^{\Delta}\right)-u\left(\bar{y}_{(k-2) \Delta}, r_{k-2}^{\Delta}\right)\right|^{2} \\
& +\frac{1}{1-\varepsilon}\left|f\left(\bar{y}_{(k-1) \Delta}, r_{k-1}^{\Delta}\right) \Delta+g\left(\bar{y}_{(k-1) \Delta}, r_{k-1}^{\Delta}\right) \Delta w_{k-1}\right|^{2} \\
\leq & \frac{2 \kappa^{2}}{\varepsilon}\left\|\bar{y}_{(k-1) \Delta}-\bar{y}_{(k-2) \Delta}\right\|^{2}+\frac{8 \kappa^{2}}{\varepsilon}\left\|\bar{y}_{(k-2) \Delta}\right\|^{2} \\
& +\frac{2}{1-\varepsilon}\left|f\left(\bar{y}_{(k-1) \Delta}, r_{k-1}^{\Delta}\right)\right|^{2} \Delta^{2}+\frac{2}{1-\varepsilon}\left|g\left(\bar{y}_{(k-1) \Delta}, r_{k-1}^{\Delta}\right) \Delta w_{k-1}\right|^{2} .
\end{aligned}
$$

\section{Consequently}

$$
\begin{aligned}
\mathbb{E}\left(\sup _{1 \leq k \leq M-1}|\bar{y}(k \Delta)-\bar{y}((k-1) \Delta)|^{2}\right) \\
\leq \frac{2 \kappa^{2}}{\varepsilon} \mathbb{E}\left(\sup _{1 \leq k \leq M-1}\left\|\bar{y}_{(k-1) \Delta}-\bar{y}_{(k-2) \Delta}\right\|^{2}\right)+\frac{8 \kappa^{2}}{\varepsilon} \mathbb{E}\left(\sup _{1 \leq k \leq M-1}\left\|\bar{y}_{(k-2) \Delta}\right\|^{2}\right) \\
\quad+\frac{2 \Delta^{2}}{1-\varepsilon} \mathbb{E}\left(\sup _{1 \leq k \leq M-1}\left|f\left(\bar{y}_{(k-1) \Delta}, \bar{r}(t)\right)\right|^{2}\right)+\frac{2}{1-\varepsilon} \mathbb{E}\left(\sup _{1 \leq k \leq M-1}\left|g\left(\bar{y}_{(k-1) \Delta}, \bar{r}(t)\right) \Delta w_{k-1}\right|^{2}\right) .
\end{aligned}
$$

We deal with these four terms, separately. By (3.21),

$$
\begin{aligned}
\mathbb{E}\left(\sup _{1 \leq k \leq M-1}\left\|\bar{y}_{(k-1) \Delta}-\bar{y}_{(k-2) \Delta}\right\|^{2}\right) \\
\quad \leq \mathbb{E}\left(\sup _{1 \leq k \leq M-1-N \leq i \leq 0} \sup _{1}|\bar{y}((k+i-1) \Delta)-\bar{y}((k-2+i) \Delta)|^{2}\right) \\
\quad \leq \mathbb{E}\left(\sup _{-N \leq k \leq M-1}|\bar{y}(k \Delta)-\bar{y}((k-1) \Delta)|^{2}\right)
\end{aligned}
$$




$$
\begin{aligned}
& \leq \mathbb{E}\left(\sup _{-N \leq k \leq 0}|\bar{y}(k \Delta)-\bar{y}((k-1) \Delta)|^{2}\right)+\mathbb{E}\left(\sup _{1 \leq k \leq M-1}|\bar{y}(k \Delta)-\bar{y}((k-1) \Delta)|^{2}\right) \\
& \leq \alpha(\Delta)+\mathbb{E}\left(\sup _{1 \leq k \leq M-1}|\bar{y}(k \Delta)-\bar{y}((k-1) \Delta)|^{2}\right) .
\end{aligned}
$$

Noting that $\mathbb{E}\left[\sup _{-\tau \leq t \leq T}|\bar{y}(t)|^{2}\right] \leq \mathbb{E}\left[\sup _{-\tau \leq t \leq T}|y(t)|^{2}\right] \leq H(2)$ (where $H(p)$ has been defined in Lemma 3.1), by Assumption 2.3 and (2.15),

$$
\begin{aligned}
& \mathbb{E}\left(\sup _{1 \leq k \leq M-1}\left|f\left(\bar{y}_{(k-1) \Delta}, r_{k-1}^{\Delta}\right)\right|^{2}\right) \\
& \leq \mathbb{E}\left(\sup _{1 \leq k \leq M-1} K\left(1+\left\|\bar{y}_{(k-1) \Delta}\right\|^{2}\right)\right) \\
& \leq K+K \mathbb{E}\left(\sup _{1 \leq k \leq M-1} \sup _{-N \leq i \leq 0}|\bar{y}((k-1+i) \Delta)|^{2}\right) \\
& \leq K+K \mathbb{E}\left(\sup _{-N \leq k \leq M-1}|\bar{y}(k \Delta)|^{2}\right) \\
& \leq K+K \mathbb{E}\left(\sup _{-\tau \leq t \leq T}|\bar{y}(t)|^{2}\right) \\
& \leq K(1+H(2)) .
\end{aligned}
$$

By the Hölder inequality, for any integer $l>1$,

$$
\begin{aligned}
\mathbb{E}\left(\sup _{1 \leq k \leq M-1}\left|g\left(\bar{y}_{(k-1) \Delta}, r_{k-1}^{\Delta}\right) \Delta w_{k-1}\right|^{2}\right) \\
\leq \mathbb{E}\left(\sup _{1 \leq k \leq M-1}\left|g\left(\bar{y}_{(k-1) \Delta}, \bar{r}(t)\right)\right|^{2} \sup _{1 \leq k \leq M-1}\left|\Delta w_{k-1}\right|^{2}\right) \\
\leq\left[\mathbb{E}\left(\sup _{1 \leq k \leq M-1}\left|g\left(\bar{y}_{(k-1) \Delta}, r_{k-1}^{\Delta}\right)\right|^{2 l /(l-1)}\right)\right]^{(l-1) / l}\left[\mathbb{E}\left(\sup _{1 \leq k \leq M-1}\left|\Delta w_{k-1}\right|^{2 l}\right)\right]^{1 / l} \\
\leq\left[\mathbb{E}\left(\sup _{0 \leq k \leq M-1}\left(K\left(1+\left\|\bar{y}_{k \Delta}\right\|^{2}\right)\right)^{l /(l-1)}\right)\right]^{(l-1) / l}\left[\mathbb{E}\left(\sum_{k=0}^{M-1}\left|\Delta w_{k}\right|^{2 l}\right)\right]^{1 / l} \\
\leq\left[K^{l /(1-l)} \mathbb{E}\left(1+\left(\sup _{0 \leq k \leq M-1}\left\|\bar{y}_{k \Delta}\right\|^{2}\right)\right)^{l /(l-1)}\right]^{(l-1) / l}\left[\left(\sum_{k=0}^{M-1} \mathbb{E}\left|\Delta w_{k}\right|^{2 l}\right)\right]^{1 / l}
\end{aligned}
$$


Journal of Applied Mathematics

$$
\begin{aligned}
& \leq\left[2^{1 /(l-1)} K^{l /(1-l)}\left(1+\mathbb{E}\left(\sup _{0 \leq k \leq M-1}\left\|\bar{y}_{k \Delta}\right\|^{2 l /(l-1)}\right)\right)\right]^{(l-1) / l}\left[\left(\sum_{k=0}^{M-1}(2 l-1) ! ! \Delta^{l}\right)\right]^{1 / l} \\
& \leq\left[2^{1 /(l-1)} K^{l /(1-l)}\left(1+H\left(\frac{2 l}{l-1}\right)\right)\right]^{(l-1) / l}\left[(2 l-1) ! ! T \Delta^{l-1}\right]^{1 / l} \\
& \leq D(l) \Delta^{(l-1) / l}
\end{aligned}
$$

where $D(l)$ is a constant dependent on $l$.

Substituting (3.25), (3.26), and (3.27) into (3.24), choosing $\varepsilon=\kappa$, and noting $\Delta \in(0,1)$, we have

$$
\begin{aligned}
& \mathbb{E}\left(\sup _{1 \leq k \leq M-1}|\bar{y}(k \Delta)-\bar{y}((k-1) \Delta)|^{2}\right) \\
& \quad \leq \frac{2 \kappa}{1-2 \kappa} \alpha(\Delta)+\frac{8 \kappa}{1-2 \kappa} H(2)+\frac{2 K(1+H(2))+2 D(l)}{(1-2 \kappa)^{2}} \Delta^{(l-1) / l}
\end{aligned}
$$

Combining (3.21) with (3.28), from (3.20), we have

$$
\begin{aligned}
\mathbb{E}\left(\sup _{0 \leq k \leq M-1}\left\|\bar{y}_{k \Delta}-\bar{y}_{(k-1) \Delta}\right\|^{2}\right) \\
\quad \leq \mathbb{E}\left(\sup _{-N \leq k \leq M-1}|\bar{y}(k \Delta)-\bar{y}((k-1) \Delta)|^{2}\right) \\
\quad \leq \mathbb{E}\left(\sup _{-N \leq k \leq 0}|\bar{y}(k \Delta)-\bar{y}((k-1) \Delta)|^{2}\right)+\mathbb{E}\left(\sup _{1 \leq k \leq M-1}|\bar{y}(k \Delta)-\bar{y}((k-1) \Delta)|^{2}\right) \\
\quad \leq \frac{1}{1-2 \kappa} \alpha(\Delta)+\frac{8 \kappa}{1-2 \kappa} H(2)+\frac{2 K(1+H(2))+2 D(l)}{(1-2 \kappa)^{2}} \Delta^{(l-1) / l},
\end{aligned}
$$

as required.

Lemma 3.3. If Assumptions 2.3-2.5 hold,

$$
\mathbb{E}\left(\sup _{0 \leq s \leq T}\left\|y_{s}-\bar{y}_{s}\right\|^{2}\right) \leq c_{2}^{\prime}+c_{2} \alpha(2 \Delta)+\bar{c}_{2}(l) \Delta^{(l-1) / l}=: \beta(\Delta),
$$

where $c_{2}, c_{2}^{\prime}$ are a constant independent of $l$ and $\Delta, \bar{c}_{2}(l)$ is a constant dependent on $l$ but independent of $\Delta$. 
Proof. Fix any $s \in[0, T]$ and $\theta \in[-\tau, 0]$. Let $k_{s} \in\{0,1,2, \ldots, M-1\}, k_{\theta} \in\{-N,-N+1, \ldots,-1\}$, and $k_{s \theta} \in\{-N,-N+1, \ldots, M-1\}$ be the integers for which $s \in\left[k_{s} \Delta,\left(k_{s}+1\right) \Delta\right], \theta \in\left[k_{\theta} \Delta,\left(k_{\theta}+\right.\right.$ 1) $\Delta]$, and $s+\theta \in\left[k_{s \theta} \Delta,\left(k_{s \theta}+1\right) \Delta\right]$, respectively. Clearly,

$$
\begin{gathered}
0 \leq s+\theta-\left(k_{s}+k_{\theta}\right) \leq 2 \Delta, \\
k_{s \theta}-\left(k_{s}+k_{\theta}\right) \in\{0,1,2\} .
\end{gathered}
$$

From (2.7),

$$
\begin{aligned}
\bar{y}_{s} & =\bar{y}_{k_{s} \Delta}(\theta) \\
& =\bar{y}\left(\left(k_{s}+k_{\theta}\right) \Delta\right)+\frac{\theta-k_{\theta} \Delta}{\Delta}\left(\bar{y}\left(\left(k_{s}+k_{\theta}+1\right) \Delta\right)-\bar{y}\left(k_{s}+k_{\theta}\right) \Delta\right),
\end{aligned}
$$

which yields

$$
\begin{aligned}
\left|y_{s}-\bar{y}_{s}\right| & =\left|y(s+\theta)-\bar{y}_{k_{s} \Delta}(\theta)\right| \\
& \leq\left|y(s+\theta)-\bar{y}\left(\left(k_{s}+k_{\theta}\right) \Delta\right)\right|+\frac{\theta-k_{\theta} \Delta}{\Delta}\left|\bar{y}\left(\left(k_{s}+k_{\theta}+1\right) \Delta\right)-\bar{y}\left(\left(k_{s}+k_{\theta}\right) \Delta\right)\right| \\
& \leq\left|y(s+\theta)-\bar{y}\left(\left(k_{s}+k_{\theta}\right) \Delta\right)\right|+\left|\bar{y}\left(\left(k_{s}+k_{\theta}+1\right) \Delta\right)-\bar{y}\left(\left(k_{s}+k_{\theta}\right) \Delta\right)\right|,
\end{aligned}
$$

so by (3.20) and Lemma 3.2, noting $\bar{y}(M \Delta)=\bar{y}((M-1) \Delta)$ from (2.15),

$$
\begin{aligned}
\mathbb{E}\left(\sup _{0 \leq s \leq T}\left\|y_{s}-\bar{y}_{s}\right\|^{2}\right) \leq & 2 \mathbb{E}\left[\sup _{0 \leq s \leq T}\left(\sup _{-\tau \leq \theta \leq 0}\left|y(s+\theta)-\bar{y}\left(\left(k_{s}+k_{\theta}\right) \Delta\right)\right|^{2}\right)\right] \\
& +2 \mathbb{E}\left[\sup _{0 \leq k_{s} \leq M-1}\left(\sup _{-N \leq k_{\theta} \leq 0}\left|\bar{y}\left(\left(k_{s}+k_{\theta}+1\right) \Delta\right)-\bar{y}\left(\left(k_{s}+k_{\theta}\right) \Delta\right)\right|^{2}\right)\right] \\
\leq & 2 \mathbb{E}\left(\sup _{-\tau \leq \theta \leq 0,0 \leq s \leq T}\left|y(s+\theta)-\bar{y}\left(\left(k_{s}+k_{\theta}\right) \Delta\right)\right|^{2}\right)+2 \gamma(\Delta) .
\end{aligned}
$$

Therefore, it is a key to compute $\mathbb{E}\left(\sup _{-\tau \leq \theta \leq 0,0 \leq s \leq T}\left|y(s+\theta)-\bar{y}\left(\left(k_{s}+k_{\theta}\right) \Delta\right)\right|^{2}\right)$. We discuss the following four possible cases.

Case $1\left(k_{s}+k_{\theta} \geq 0\right)$. We again divide this case into three possible subcases according to $k_{s \theta}-\left(k_{s}+k_{\theta}\right) \in\{0,1,2\}$. 
Subcase $1\left(k_{s \theta}-\left(k_{s}+k_{\theta}\right)=0\right)$. From $(2.13)$,

$$
\begin{aligned}
y(s+\theta)-\bar{y}\left(\left(k_{s}+k_{\theta}\right) \Delta\right)= & u\left(\bar{y}_{\left(k_{s \theta}-1\right) \Delta}+\frac{s+\theta-k_{s \theta} \Delta}{\Delta}\left(\bar{y}_{k_{s \theta} \Delta}-\bar{y}_{\left(k_{s \theta}-1\right) \Delta}\right), r_{k_{s \theta}}^{\Delta}\right) \\
& -u\left(\bar{y}_{\left(k_{s \theta}-1\right) \Delta}, r_{k_{s \theta-1}}^{\Delta}\right)+\int_{k_{s \theta} \Delta}^{s+\theta} f\left(\bar{y}_{v}, \bar{r}(v)\right) d v \\
& +\int_{k_{s \theta} \Delta}^{s+\theta} g\left(\bar{y}_{v}, \bar{r}(v)\right) d w(v),
\end{aligned}
$$

which yields

$$
\begin{aligned}
& \mathbb{E}\left(\sup _{-\tau \leq \theta \leq 0,0 \leq s \leq T, k_{s}+k_{\theta} \geq 0}\left|y(s+\theta)-\bar{y}\left(\left(k_{s}+k_{\theta}\right) \Delta\right)\right|^{2}\right) \\
& \leq 3 \mathbb{E}\left(\sup _{-\tau \leq \theta \leq 0,0 \leq s \leq T, k_{s \theta} \geq 0} \mid u\left(\bar{y}_{\left(k_{s \theta}-1\right) \Delta}+\frac{s+\theta-k_{s \theta} \Delta}{\Delta}\left(\bar{y}_{k_{s \theta} \Delta}-\bar{y}_{\left(k_{s \theta}-1\right) \Delta}, r_{k_{s \theta}}^{\Delta}\right)\right)\right. \\
& \left.-\left.u\left(\bar{y}_{\left(k_{s \theta}-1\right) \Delta}, r_{k_{s \theta-1}}^{\Delta}\right)\right|^{2}\right)+3 \mathbb{E}\left(\sup _{-\tau \leq \theta \leq 0,0 \leq s \leq T, k_{s \theta} \geq 0}\left|\int_{k_{s \theta} \Delta}^{s+\theta} f\left(\bar{y}_{r}, \bar{r}(r)\right) d r\right|^{2}\right) \\
& +3 \mathbb{E}\left(\sup _{-\tau \leq \theta \leq 0,0 \leq s \leq T, k_{s \theta} \geq 0}\left|\int_{k_{s \theta} \Delta}^{s+\theta} g\left(\bar{y}_{r}, \bar{r}(r)\right) d w(r)\right|^{2}\right) .
\end{aligned}
$$

From Assumption 2.4, (3.24), (3.26), and Lemma 3.2, we have

$$
\begin{aligned}
& \mathbb{E}\left(\sup _{-\tau \leq \theta \leq 0,0 \leq s \leq T, k_{s \theta} \geq 0}\left|u\left(\bar{y}_{\left(k_{s \theta}-1\right) \Delta}+\frac{s+\theta-k_{s \theta} \Delta}{\Delta}\left(\bar{y}_{k_{s \theta} \Delta}-\bar{y}_{\left(k_{s \theta}-1\right) \Delta}, r_{k_{s \theta}}^{\Delta}\right)\right)-u\left(\bar{y}_{\left(k_{s \theta}-1\right) \Delta}, r_{s_{s \theta-1}}^{\Delta}\right)\right|^{2}\right) \\
& \quad \leq 2 \kappa^{2} \mathbb{E}\left(\sup _{-\tau \leq \theta \leq 0,0 \leq s \leq T, k_{s \theta} \geq 0}\left\|\frac{s+\theta-k_{s \theta} \Delta}{\Delta}\left(\bar{y}_{k_{s \theta} \Delta}-\bar{y}_{\left(k_{s \theta}-1\right) \Delta}\right)\right\|^{2}\right)+8 \kappa^{2} H(2) \\
& \quad \leq 2 \kappa^{2} \mathbb{E}\left(\sup _{-\tau \leq \theta \leq 0,0 \leq s \leq T, k_{s \theta} \geq 0}\left\|\left(\bar{y}_{k_{s \theta} \Delta}-\bar{y}_{\left(k_{s \theta}-1\right) \Delta}\right)\right\|^{2}\right)+8 \kappa^{2} H(2) \\
& \quad \leq 2 \kappa^{2} \mathbb{E}\left(\sup _{0 \leq k_{s \theta} \leq M-1}\left\|\left(\bar{y}_{k_{s \theta} \Delta}-\bar{y}_{\left(k_{s \theta}-1\right) \Delta}\right)\right\|^{2}\right)+8 \kappa^{2} H(2) \\
& \leq 2 \kappa^{2} \gamma(\Delta)+8 \kappa^{2} H(2) .
\end{aligned}
$$


By the Hölder inequality, Assumption 2.3, and Lemma 3.1,

$$
\begin{aligned}
& \mathbb{E}\left(\sup _{-\tau \leq \theta \leq 0,0 \leq s \leq T, k_{s \theta} \geq 0}\left|\int_{k_{s \theta} \Delta}^{s+\theta} f\left(\bar{y}_{r}, \bar{r}(r)\right) d r\right|^{2}\right) \\
& \leq \Delta \mathbb{E}\left(\sup _{-\tau \leq \theta \leq 0,0 \leq s \leq T, k_{s \theta} \geq 0} \int_{k_{s \theta} \Delta}^{s+\theta}\left|f\left(\bar{y}_{r}, \bar{r}(r)\right)\right|^{2} d r\right) \\
& \leq K \Delta \mathbb{E}\left(\sup _{-\tau \leq \theta \leq 0,0 \leq s \leq T, k_{s \theta} \geq 0} \int_{k_{s \theta} \Delta}^{s+\theta}\left(1+\left\|\bar{y}_{r}\right\|^{2}\right) d r\right) \\
& \leq K \Delta \mathbb{E}\left[\int_{0}^{T}\left(1+\sup _{0 \leq r \leq T}\left\|\bar{y}_{r}\right\|^{2}\right) d r\right] \\
& \leq K \Delta \int_{0}^{T}\left[1+\mathbb{E}\left(\left.\sup _{-\tau \leq t \leq T} y(t)\right|^{2}\right)\right] d r \\
& \leq K \Delta \int_{0}^{T}[1+H(2)] d r \\
& \leq K T[1+H(2)] \Delta .
\end{aligned}
$$

Setting $v=s+\theta$ and $k_{v}=k_{s \theta}$ and applying the Hölder inequality yield

$$
\begin{aligned}
\mathbb{E}\left(\sup _{-\tau \leq \theta \leq 0,0 \leq s \leq T, k_{s \theta} \geq 0}\left|\int_{k_{s \theta} \Delta}^{s+\theta} g\left(\bar{y}_{r}, \bar{r}(r)\right) d w(r)\right|^{2}\right) \\
=\mathbb{E}\left(\sup _{0 \leq v \leq T, 0 \leq k_{v} \leq M-1}\left|g\left(\bar{y}_{k_{v} \Delta}, \bar{r}(v)\right)\left(w(v)-w\left(k_{v} \Delta\right)\right)\right|^{2}\right) \\
\leq\left[\mathbb{E}\left(\sup _{0 \leq v \leq T, 0 \leq k_{v} \leq M-1}\left|g\left(\bar{y}_{k_{v} \Delta}, \bar{r}(v)\right)\right|^{2 l /(l-1)}\right)\right]^{(l-1) / l} \\
\times\left[\mathbb{E}\left(\sup _{0 \leq v \leq T, 0 \leq k_{v} \leq M-1}\left|w(v)-w\left(k_{v} \Delta\right)\right|^{2 l}\right)\right]^{1 / l} .
\end{aligned}
$$

The Doob martingale inequality gives

$$
\begin{aligned}
\mathbb{E}\left(\sup _{0 \leq v \leq T, 0 \leq k_{v} \leq M-1}\left|w(v)-w\left(k_{v} \Delta\right)\right|^{2 l}\right) \\
=\mathbb{E}\left(\sup _{0 \leq k_{v} \leq M-1}\left(\sup _{k_{v} \Delta \leq v \leq\left(k_{v}+1\right) \Delta}\left|w(v)-w\left(k_{v} \Delta\right)\right|^{2 l}\right)\right)
\end{aligned}
$$


Journal of Applied Mathematics

$$
\begin{aligned}
& \leq \mathbb{E}\left(\sum_{k_{v}=0}^{M-1}\left(\sup _{k_{v} \Delta \leq v \leq\left(k_{v}+1\right) \Delta}\left|w(v)-w\left(k_{v} \Delta\right)\right|^{2 l}\right)\right) \\
& =\sum_{k_{v}=0}^{M-1} \mathbb{E}\left(\sup _{k_{v} \Delta \leq v \leq\left(k_{v}+1\right) \Delta}\left|w(v)-w\left(k_{v} \Delta\right)\right|^{2 l}\right) \\
& \leq\left(\frac{2 l}{2 l-1}\right)^{2 l} \sum_{k_{v}=0}^{2-1} \mathbb{E}\left|w\left(\left(k_{v}+1\right) \Delta\right)-w\left(k_{v} \Delta\right)\right|^{2 l} .
\end{aligned}
$$

By (3.27), we therefore have

$$
\begin{aligned}
\mathbb{E}\left(\sup _{-\tau \leq \theta \leq 0,0 \leq s \leq T, k_{s \theta} \geq 0}\left|\int_{k_{s \theta} \Delta}^{s+\theta} g\left(\bar{y}_{r}, \bar{r}(r)\right) d w(r)\right|^{2}\right) \\
\leq\left(\frac{2 l}{2 l-1}\right)^{2}\left[\mathbb{E}\left(\sup _{0 \leq k_{v} \leq M-1}\left|g\left(\bar{y}_{k_{v} \Delta}, \bar{r}(v)\right)\right|^{2 l /(l-1)}\right)\right]^{(l-1) / l} \\
\times\left[\sum_{k_{v}=0}^{M-1} \mathbb{E}\left|w\left(\left(k_{v}+1\right) \Delta\right)-w\left(k_{v} \Delta\right)\right|^{2 l}\right]^{1 / l} \\
\leq\left(\frac{2 l}{2 l-1}\right)^{2} D(l) \Delta^{(l-1) / l} .
\end{aligned}
$$

Substituting (3.37), (3.38), and (3.41) into (3.36) and noting $\Delta \in(0,1)$ give

$$
\begin{aligned}
& \mathbb{E}\left(\sup _{-\tau \leq \theta \leq 0,0 \leq s \leq T, k_{s}+k_{\theta} \geq 0}\left|y(s+\theta)-\bar{y}\left(\left(k_{s}+k_{\theta}\right) \Delta\right)\right|^{2}\right) \\
& \quad \leq 6 \kappa^{2} \gamma(\Delta)+24 \kappa^{2} H(2)+3\left(K T(1+H(2))+\left(\frac{2 l}{2 l-1}\right)^{2} D(l)\right) \Delta^{(l-1) / l} \\
& =: 6 \kappa^{2} \gamma(\Delta)+24 \kappa^{2} H(2)+c_{1}(l) \Delta^{(l-1) / l} .
\end{aligned}
$$

Subcase2 $\left(k_{s \theta}-\left(k_{s}+k_{\theta}\right)=1\right)$. From (2.13),

$$
\begin{aligned}
y(s+\theta)-\bar{y}\left(\left(k_{s}+k_{\theta}\right) \Delta\right)= & y\left(k_{s \theta} \Delta\right)-\bar{y}\left(\left(k_{s}+k_{\theta}\right) \Delta\right)+y(s+\theta)-\bar{y}\left(k_{s \theta} \Delta\right) \\
\leq & u\left(\bar{y}_{\left(k_{s}+k_{\theta}\right) \Delta}, r_{k_{s}+k_{\theta}}^{\Delta}\right)-u\left(\bar{y}_{\left(k_{s}+k_{\theta}-1\right) \Delta}, r_{k_{s}+k_{\theta}-1}^{\Delta}\right)+f\left(\bar{y}_{\left(k_{s}+k_{\theta}\right) \Delta}, r_{k_{s}+k_{\theta}}^{\Delta}\right) \Delta \\
& +g\left(\bar{y}_{\left(k_{s}+k_{\theta}\right) \Delta}, r_{k_{s}+k_{\theta}}^{\Delta}\right) \Delta w_{k_{s}+k_{\theta}}+y(s+\theta)-\bar{y}\left(k_{s \theta} \Delta\right),
\end{aligned}
$$


so we have

$$
\begin{gathered}
\mathbb{E}\left(\sup _{-\tau \leq \theta \leq 0,0 \leq s \leq T, k_{s}+k_{\theta} \geq 0}\left|y(s+\theta)-\bar{y}\left(\left(k_{s}+k_{\theta}\right) \Delta\right)\right|^{2}\right) \\
\leq 4\left\{\mathbb{E}\left(\sup _{-\tau \leq \theta \leq 0,0 \leq s \leq T, k_{s}+k_{\theta} \geq 0}\left|u\left(\bar{y}_{\left(k_{s}+k_{\theta}\right) \Delta}, r_{k_{s}+k_{\theta}}^{\Delta}\right)-u\left(\bar{y}_{\left(k_{s}+k_{\theta}-1\right) \Delta}, r_{k_{s}+k_{\theta}-1}^{\Delta}\right)\right|^{2}\right)\right. \\
+\mathbb{E}\left(\sup _{-\tau \leq \theta \leq 0,0 \leq s \leq T, k_{s}+k_{\theta} \geq 0}\left|f\left(\bar{y}_{\left(k_{s}+k_{\theta}\right) \Delta}, r_{k_{s}+k_{\theta}}^{\Delta}\right) \Delta\right|^{2}\right) \\
+\mathbb{E}\left(\sup _{-\tau \leq \theta \leq 0,0 \leq s \leq T, k_{s}+k_{\theta} \geq 0}\left|g\left(\bar{y}_{\left(k_{s}+k_{\theta}\right) \Delta}, r_{k_{s}+k_{\theta}}^{\Delta}\right) \Delta w_{k_{s}+k_{\theta}}\right|^{2}\right) \\
\left.+\mathbb{E}\left(\sup _{-\tau \leq \theta \leq 0,0 \leq s \leq T, k_{s}+k_{\theta} \geq 0}\left|y(s+\theta)-\bar{y}\left(k_{s \theta} \Delta\right)\right|^{2}\right)\right\} .
\end{gathered}
$$

Since

$$
\mathbb{E}\left(\sup _{-\tau \leq \theta \leq 0,0 \leq s \leq T, k_{s}+k_{\theta} \geq 0}\left|u\left(\bar{y}_{\left(k_{s}+k_{\theta}\right) \Delta}, r_{k_{s}+k_{\theta}}^{\Delta}\right)-u\left(\bar{y}_{\left(k_{s}+k_{\theta}-1\right) \Delta}, r_{k_{s}+k_{\theta}-1}^{\Delta}\right)\right|^{2}\right) \leq 2 \kappa^{2} \gamma(\Delta)+8 \kappa^{2} H(2)
$$

from (3.26), (3.27), and the Subcase 1 , noting $\Delta \in(0,1)$, we have

$$
\begin{aligned}
\mathbb{E}\left(\sup _{-\tau \leq \theta \leq 0,0 \leq s \leq T, k_{s}+k_{\theta} \geq 0}\left|y(s+\theta)-\bar{y}\left(\left(k_{s}+k_{\theta}\right) \Delta\right)\right|^{2}\right) \\
\leq 4\left\{2 \kappa^{2} \gamma(\Delta)+8 \kappa^{2} H(2)+K[1+H(2)] \Delta^{2}+D(l) \Delta^{(l-1) / l}\right. \\
\left.+3\left(2 \kappa^{2} \gamma(\Delta)+8 \kappa^{2} H(2)\right)(\Delta)+c_{1}(l) \Delta^{(l-1) / l}\right\} \\
=: 32 \kappa^{2} \gamma(\Delta)+128 \kappa^{2} H(2)+c_{2}(l) \Delta^{(l-1) / l} .
\end{aligned}
$$

Subcase $3\left(k_{s \theta}-\left(k_{s}+k_{\theta}\right)=2\right)$. From (2.13), we have

$$
y(s+\theta)-\bar{y}\left(\left(k_{s}+k_{\theta}\right) \Delta\right)=y(s+\theta)-y\left(\left(k_{s \theta}-1\right) \Delta\right)+y\left(\left(k_{s \theta}-1\right) \Delta\right)-\bar{y}\left(\left(k_{s}+k_{\theta}\right) \Delta\right),
$$


Journal of Applied Mathematics

so from the Subcase 2, we have

$$
\begin{aligned}
\mathbb{E}\left(\sup _{-\tau \leq \theta \leq 0,0 \leq s \leq T, k_{s}+k_{\theta} \geq 0}\left|y(s+\theta)-\bar{y}\left(\left(k_{s}+k_{\theta}\right) \Delta\right)\right|^{2}\right) \\
\leq 2 \mathbb{E}\left(\sup _{-\tau \leq \theta \leq 0,0 \leq s \leq T, k_{s}+k_{\theta} \geq 0}\left|y(s+\theta)-\bar{y}\left(\left(k_{s \theta}-1\right) \Delta\right)\right|^{2}\right) \\
+2 \mathbb{E}\left(\sup _{-\tau \leq \theta \leq 0,0 \leq s \leq T, k_{s}+k_{\theta} \geq 0}\left|y\left(\left(k_{s \theta}-1\right) \Delta\right)-\bar{y}\left(\left(k_{s}+k_{\theta}\right) \Delta\right)\right|^{2}\right) \\
\leq 2\left[32 \kappa^{2} \gamma(\Delta)+128 \kappa^{2} H(2)+c_{2}(l) \Delta^{(l-1) / l}\right] \\
+2\left[2 \kappa^{2} \gamma(\Delta)+8 \kappa^{2} H(2)+K[1+H(2)] \Delta^{2}+D(l) \Delta^{(l-1) / l}\right] \\
=: 68 \kappa^{2} \gamma(\Delta)+272 \kappa^{2} H(2)+c_{3}(l) \Delta^{(l-1) / l} .
\end{aligned}
$$

From these three subcases, we have

$$
\begin{aligned}
& \mathbb{E}\left(\sup _{-\tau \leq \theta \leq 0,0 \leq s \leq T, k_{s}+k_{\theta} \geq 0}\left|y(s+\theta)-\bar{y}\left(\left(k_{s}+k_{\theta}\right) \Delta\right)\right|^{2}\right) \\
& \quad \leq 106 \kappa^{2} \gamma(\Delta)+424 \kappa^{2} H(2)+\left[c_{1}(l)+c_{2}(l)+c_{3}(l)\right] \Delta^{(l-1) / l} .
\end{aligned}
$$

Case $2\left(k_{s}+k_{\theta}=-1\right.$ and $\left.0 \leq s+\theta \leq \Delta\right)$. In this case, applying Assumption 2.5 and case 1 , we have

$$
\begin{aligned}
& \mathbb{E}\left(\sup _{-\tau \leq \theta \leq 0,0 \leq s \leq T}\left|y(s+\theta)-\bar{y}\left(\left(k_{s}+k_{\theta}\right) \Delta\right)\right|^{2}\right) \\
& \quad \leq 2 \mathbb{E}\left(\sup _{-\tau \leq \theta \leq 0,0 \leq s \leq T}|y(s+\theta)-\bar{y}(0)|^{2}\right)+2 E|y(0)-y(-\Delta)|^{2} \\
& \quad \leq 212 \kappa^{2} \gamma(\Delta)+848 \kappa^{2} H(2)+2\left[c_{1}(l)+c_{2}(l)+c_{3}(l)\right] \Delta^{(l-1) / l}+2 \alpha(\Delta) .
\end{aligned}
$$

Case $3\left(k_{s}+k_{\theta}=-1\right.$ and $\left.-\Delta \leq s+\theta \leq 0\right)$. In this case, using Assumption 2.5,

$$
\mathbb{E}\left(\sup _{-\tau \leq \theta \leq 0,0 \leq s \leq T}\left|y(s+\theta)-\bar{y}\left(\left(k_{s}+k_{\theta}\right) \Delta\right)\right|^{2}\right) \leq \alpha(\Delta) .
$$


Case $4\left(k_{s}+k_{\theta} \leq-2\right)$. In this case, $s+\theta \leq 0$, so using Assumption 2.5,

$$
\mathbb{E}\left(\sup _{-\tau \leq \theta \leq 0,0 \leq s \leq T}\left|y(s+\theta)-\bar{y}\left(\left(k_{s}+k_{\theta}\right) \Delta\right)\right|^{2}\right) \leq \alpha(2 \Delta) .
$$

Substituting these four cases into (3.34) and noting the expression of $\gamma(\Delta)$, there exist $c_{2}, c_{2}^{\prime}$, and $\bar{c}_{2}(l)$ such that

$$
\mathbb{E}\left(\sup _{0 \leq s \leq T}\left\|y_{s}-\bar{y}_{s}\right\|^{2}\right) \leq c_{2} \alpha(2 \Delta)+c_{2}^{\prime}+\bar{c}_{2}(l) \Delta^{(l-1) / l},
$$

This proof is complete.

Remark 3.4. It should be pointed out that much simpler proofs of Lemmas 3.2 and 3.3 can be obtained by choosing $l=2$ if we only want to prove Theorem 2.7. However, here we choose $l>1$ to control the stochastic terms $\beta(\Delta)$ and $\gamma(\Delta)$ by $\Delta^{(l-1) / l}$ in Section 3, which will be used to show the order of the strong convergence.

Lemma 3.5. If Assumption 2.4 holds,

$$
\mathbb{E}\left(\sup _{0 \leq t \leq T}\left|u\left(\bar{y}_{t}, \bar{r}(t)\right)-u\left(\bar{y}_{t}, r(t)\right)\right|^{2}\right) \leq 8 \kappa^{2} H(2) L \Delta:=\zeta \Delta
$$

where $L$ is a positive constant independent of $\Delta$.

Proof. Let $n=[T / \Delta]$, the integer part of $T / \Delta$, and $1_{G}$ be the indication function of the set $G$. Then, by Assumption 2.4 and Lemma 3.1, we obtain

$$
\begin{aligned}
& \mathbb{E}\left(\sup _{0 \leq t \leq T}\left|u\left(\bar{y}_{t}, \bar{r}(t)\right)-u\left(\bar{y}_{t}, r(t)\right)\right|^{2}\right) \\
& \quad \leq \max _{0 \leq k \leq n} \mathbb{E}\left(\sup _{s \in\left[t_{k}, t_{k+1}\right)}\left|u\left(\bar{y}_{s}, \bar{r}(s)\right)-u\left(\bar{y}_{s}, r(s)\right)\right|^{2}\right) \\
& \quad \leq 2 \max _{0 \leq k \leq n} \mathbb{E}\left(\sup _{s \in\left[t_{k}, t_{k+1}\right)}\left|u\left(\bar{y}_{s}, \bar{r}(s)\right)-u\left(\bar{y}_{s}, r(s)\right)\right|^{2} 1_{\left\{r(s) \neq r\left(t_{k}\right)\right\}}\right) \\
& \quad \leq 4 \max _{0 \leq k \leq n} \mathbb{E}\left(\sup _{s \in\left[t_{k}, t_{k+1}\right)}\left(\left|u\left(\bar{y}_{s}, \bar{r}(s)\right)\right|^{2}+\left|u\left(\bar{y}_{s}, r(s)\right)\right|^{2}\right) 1_{\left\{r(s) \neq r\left(t_{k}\right)\right\}}\right) \\
& \quad \leq 8 \kappa^{2} \max _{0 \leq k \leq n}\left(\mathbb{E}\left(\sup _{0 \leq t \leq T}\left\|\bar{y}_{t}\right\|^{2}\right)\right) \mathbb{E}\left(1_{\left\{r(s) \neq r\left(t_{k}\right)\right\}}\right)
\end{aligned}
$$


Journal of Applied Mathematics

$$
\begin{aligned}
& \leq 8 \kappa^{2} H(2) \mathbb{E}\left(1_{\left\{r(s) \neq r\left(t_{k}\right)\right\}}\right) \\
& =8 \kappa^{2} H(2) \mathbb{E}\left[\mathbb{E}\left(1_{\left\{r(s) \neq r\left(t_{k}\right)\right\}} \mid\left(t_{k}\right)\right)\right],
\end{aligned}
$$

where in the last step we use the fact that $\bar{y}_{t_{k}}$ and $1_{\left\{r(s) \neq r\left(t_{k}\right)\right\}}$ are conditionally independent with respect to the $\sigma$-algebra generated by $r\left(t_{k}\right)$. But, by the Markov property,

$$
\begin{aligned}
\mathbb{E}\left(1_{\left\{r(s) \neq r\left(t_{k}\right)\right\}} \mid r\left(t_{k}\right)\right) & =\sum_{i \in S} 1_{\left\{r\left(t_{k}\right)=i\right\}} P\left(r(s) \neq i \mid r\left(t_{k}\right)=i\right) \\
& =\sum_{i \in S} 1_{\left\{r\left(t_{k}\right)=i\right\}} \sum_{j \neq i}\left(\gamma_{i j}\left(s-t_{k}\right)+o\left(s-t_{k}\right)\right) \\
& \leq \sum_{i \in S} 1_{\left\{r\left(t_{k}\right)=i\right\}}\left(\max _{1 \leq i \leq N}\left(-\gamma_{i j}\right) \Delta+o(\Delta)\right) \\
& \leq L \Delta,
\end{aligned}
$$

where $L$ is a positive constant independent of $\Delta$. Then

$$
\mathbb{E}\left(\sup _{0 \leq t \leq T}\left|u\left(\bar{y}_{t}, \bar{r}(t)\right)-u\left(\bar{y}_{t}, r(t)\right)\right|^{2}\right) \leq 8 \kappa^{2} H(2) L \Delta .
$$

This proof is complete.

Lemma 3.6. If Assumption 2.3 holds, there is a constant $C$, which is independent of $\Delta$ such that

$$
\begin{aligned}
& \mathbb{E} \int_{0}^{T}\left|f\left(\bar{y}_{s}, r(s)\right)-f\left(\bar{y}_{s}, \bar{r}(s)\right)\right|^{2} d s \leq C \Delta, \\
& \mathbb{E} \int_{0}^{T}\left|g\left(\bar{y}_{s}, r(s)\right)-g\left(\bar{y}_{s}, \bar{r}(s)\right)\right|^{2} d s \leq C \Delta .
\end{aligned}
$$

Proof. Let $n=[T / \Delta]$, the integer part of $T / \Delta$. Then

$$
\mathbb{E} \int_{0}^{T}\left|f\left(\bar{y}_{s^{\prime}}, r(s)\right)-f\left(\bar{y}_{s^{\prime}}, \bar{r}(s)\right)\right|^{2} d s=\sum_{k=0}^{n} \mathbb{E} \int_{t_{k}}^{t_{k+1}}\left|f\left(\bar{y}_{t_{k^{\prime}}}, r(s)\right)-f\left(\bar{y}_{t_{k}}, r\left(t_{k}\right)\right)\right|^{2} d s,
$$


with $t_{n+1}$ being $T$. Let $1_{G}$ be the indication function of the set $G$. Moreover, in what follows, $C$ is a generic positive constant independent of $\Delta$, whose values may vary from line to line. With these notations we derive, using Assumption 2.3, that

$$
\begin{aligned}
& \mathbb{E} \int_{t_{k}}^{t_{k+1}}\left|f\left(\bar{y}_{t_{k}}, r(s)\right)-f\left(\bar{y}_{t_{k}}, r\left(t_{k}\right)\right)\right|^{2} d s \\
& \quad \leq 2 \mathbb{E} \int_{t_{k}}^{t_{k+1}}\left[\left|f\left(\bar{y}_{t_{k}}, r(s)\right)\right|^{2}+\left|f\left(\bar{y}_{t_{k}}, r\left(t_{k}\right)\right)\right|^{2}\right] 1_{\left\{r(s) \neq r\left(t_{k}\right)\right\}} d s \\
& \leq C \mathbb{E} \int_{t_{k}}^{t_{k+1}}\left(1+\left\|\bar{y}_{t_{k}}\right\|^{2}\right) 1_{\left\{r(s) \neq r\left(t_{k}\right)\right\}} d s \\
& \quad \leq C \int_{t_{k}}^{t_{k+1}} \mathbb{E}\left[\mathbb{E}\left[\left(1+\left\|\bar{y}_{t_{k}}\right\|^{2}\right) 1_{\left\{r(s) \neq r\left(t_{k}\right)\right\}} \mid r\left(t_{k}\right)\right]\right] d s \\
& \quad \leq C \int_{t_{k}}^{t_{k+1}} \mathbb{E}\left[\mathbb{E}\left[\left(1+\left\|\bar{y}_{t_{k}}\right\|^{2}\right) \mid r\left(t_{k}\right)\right] \mathbb{E}\left[1_{\left\{r(s) \neq r\left(t_{k}\right)\right\}} \mid r\left(t_{k}\right)\right]\right] d s,
\end{aligned}
$$

where in the last step we use the fact that $\bar{y}_{t_{k}}$ and $1_{\left\{r(s) \neq r\left(t_{k}\right)\right\}}$ are conditionally independent with respect to the $\sigma$-algebra generated by $r\left(t_{k}\right)$. But, by the Markov property,

$$
\begin{aligned}
\mathbb{E}\left[1_{\left\{r(s) \neq r\left(t_{k}\right)\right\}} \mid r\left(t_{k}\right)\right] & =\sum_{i \in S} 1_{\left\{r\left(t_{k}\right)=i\right\}} P\left(r(s) \neq i \mid r\left(t_{k}\right)=i\right) \\
& =\sum_{i \in S} 1_{\left\{r\left(t_{k}\right)=i\right\}} \sum_{j \neq i}\left(\gamma_{i j}\left(s-t_{k}\right)+o\left(s-t_{k}\right)\right) \\
& \leq \sum_{i \in S} 1_{\left\{r\left(t_{k}\right)=i\right\}}\left(\max _{1 \leq i \leq N}\left(-\gamma_{i j}\right) \Delta+o(\Delta)\right) \\
& \leq C \Delta .
\end{aligned}
$$

So, by Lemma 3.1,

$$
\begin{aligned}
\mathbb{E} \int_{t_{k}}^{t_{k+1}}\left|f\left(\bar{y}_{t_{k}}, r(s)\right)-f\left(\bar{y}_{t_{k}}, r\left(t_{k}\right)\right)\right|^{2} d s & \leq C \Delta \int_{t_{k}}^{t_{k+1}}\left(1+\mathbb{E}\left\|\bar{y}_{t_{k}}\right\|^{2}\right) d s \\
& \leq C \Delta^{2} .
\end{aligned}
$$

Therefore

$$
\mathbb{E} \int_{0}^{T}\left|f\left(\bar{y}_{s}, r(s)\right)-f\left(\bar{y}_{s}, \bar{r}(s)\right)\right|^{2} d s \leq C \Delta
$$

Similarly, we can show (3.59). 


\section{Convergence of the EM Methods}

In this section we will use the lemmas above to prove Theorem 2.7. From Lemma 3.1 and Theorem 2.6, there exists a positive constant $\widetilde{H}$ such that

$$
\mathbb{E}\left(\sup _{-\tau \leq t \leq T}|x(t)|^{p}\right) \vee \mathbb{E}\left(\sup _{-\tau \leq t \leq T}|y(t)|^{p}\right) \leq \widetilde{H}
$$

Let $j$ be a sufficient large integer. Define the stopping times

$$
u_{j}:=\inf \left\{t \geq 0:\left\|x_{t}\right\| \geq j\right\}, \quad v_{j}:=\inf \left\{t \geq 0:\left\|y_{t}\right\| \geq j\right\}, \quad \rho_{j}:=u_{j} \wedge v_{j}
$$

where we set $\inf \emptyset=\infty$ as usual. Let

$$
e(t):=x(t)-y(t)
$$

Obviously,

$$
\mathbb{E}\left(\sup _{0 \leq t \leq T}|e(t)|^{2}\right)=\mathbb{E}\left(\sup _{0 \leq t \leq T}|e(t)|^{2} 1_{\left\{u_{j}>T, v_{j}>T\right\}}\right)+\mathbb{E}\left(\sup _{0 \leq t \leq T}|e(t)|^{2} 1_{\left\{u_{j} \leq T \text { or } v_{j} \leq T\right\}}\right) .
$$

Recall the following elementary inequality:

$$
a^{\gamma} b^{1-\gamma} \leq \gamma a+(1-\gamma) b, \quad \forall a, b>0, \gamma \in[0,1]
$$

We thus have, for any $\delta>0$,

$$
\begin{aligned}
\mathbb{E}\left(\sup _{0 \leq t \leq T}|e(t)|^{2} 1_{\left\{u_{j} \leq T \text { or } v_{j} \leq T\right\}}\right) & \leq \mathbb{E}\left[\left(\delta \sup _{\{0 \leq t \leq T\}}|e(t)|^{p}\right)^{2 / p}\left(\delta^{-2 /(p-2)} 1_{\left\{u_{j} \leq T \text { or } v_{j} \leq T\right\}}\right)^{(p-2) / p}\right] \\
& \leq \frac{2 \delta}{p} \mathbb{E}\left(\sup _{0 \leq t \leq T}|e(t)|^{p}\right)+\frac{p-2}{p \delta^{2 /(p-2)}} \mathbb{P}\left(u_{j} \leq T \text { or } v_{j} \leq T\right) .
\end{aligned}
$$

Hence

$$
\begin{aligned}
\mathbb{E}\left(\sup _{0 \leq t \leq T}|e(t)|^{2}\right) \leq & \mathbb{E}\left(\sup _{0 \leq t \leq T}|e(t)|^{2} 1_{\left\{\rho_{j}>T\right\}}\right)+\frac{2 \delta}{p} \mathbb{E}\left(\sup _{0 \leq t \leq T}|e(t)|^{p}\right) \\
& +\frac{p-2}{p \delta^{2 /(p-2)}} \mathbb{P}\left(u_{j} \leq T \text { or } v_{j} \leq T\right) .
\end{aligned}
$$


Now,

$$
\begin{aligned}
\mathbb{P}\left(u_{j} \leq T\right) & \leq \mathbb{E}\left(1_{\left\{u_{j} \leq T\right\}} \frac{\left\|x_{t}\right\|^{p}}{j^{p}}\right) \\
& \leq \frac{1}{j^{p}} \mathbb{E}\left(\sup _{-\tau \leq t \leq T}|x(t)|^{p}\right) \\
& \leq \frac{\widetilde{H}}{j^{p}} .
\end{aligned}
$$

Similarly,

$$
\mathbb{P}\left(v_{j} \leq T\right) \leq \frac{\widetilde{H}}{j^{p}} .
$$

Thus

$$
\begin{aligned}
\mathbb{P}\left(v_{j} \leq T \text { or } u_{j} \leq T\right) & \leq \mathbb{P}\left(v_{j} \leq T\right)+\mathbb{P}\left(u_{j} \leq T\right) \\
& \leq \frac{2 \widetilde{H}}{j^{p}} .
\end{aligned}
$$

We also have

$$
\begin{aligned}
\mathbb{E}\left(\sup _{0 \leq t \leq T}|e(t)|^{p}\right) & \leq 2^{p-1} \mathbb{E}\left(\sup _{0 \leq t \leq T}\left(\left|x_{t}\right|^{p}+\left|y_{t}\right|^{p}\right)\right) \\
& \leq 2^{p} \widetilde{H}
\end{aligned}
$$

Moreover,

$$
\begin{aligned}
\mathbb{E}\left(\sup _{0 \leq t \leq T}|e(t)|^{2} 1_{\left\{\rho_{j}>T\right\}}\right) & =\mathbb{E}\left(\sup _{0 \leq t \leq T}\left|e\left(t \wedge \rho_{j}\right)\right|^{2} 1_{\left\{\rho_{j}>T\right\}}\right) \\
& \leq \mathbb{E}\left(\sup _{0 \leq t \leq T}\left|e\left(t \wedge \rho_{j}\right)\right|^{2}\right) .
\end{aligned}
$$

Using these bounds in (4.7) yields

$$
\mathbb{E}\left(\sup _{0 \leq t \leq T}|e(t)|^{2}\right) \leq \mathbb{E}\left(\sup _{0 \leq t \leq T}\left|e\left(t \wedge \rho_{j}\right)\right|^{2}\right)+\frac{2^{p+1} \delta \widetilde{H}}{p}+\frac{2(p-2) \widetilde{H}}{p \delta^{2 /(p-2)} j^{p}}
$$


Journal of Applied Mathematics

Setting $v:=t \wedge \rho_{j}$ and for any $\varepsilon \in(0,1)$, by the Hölder inequality, when $v \in[k \Delta,(k+1) \Delta]$, for $k=0,1,2, \ldots, M-1$,

$$
\begin{aligned}
|e(v)|^{2}= & |x(v)-y(v)|^{2} \\
\leq & \mid u\left(x_{v}, r(v)\right)-u\left(\bar{y}_{(k-1) \Delta}+\frac{(v-k \Delta)}{\Delta}\left(\bar{y}_{k \Delta}-\bar{y}_{(k-1) \Delta}\right), r_{k}^{\Delta}\right) \\
& +\int_{0}^{v}\left[f\left(x_{s}, r(s)\right)-f\left(\bar{y}_{s}, \bar{r}(s)\right)\right] d s+\left.\int_{0}^{v}\left[g\left(x_{s}, r(s)\right)-g\left(\bar{y}_{s}, \bar{r}(s)\right)\right] d w(s)\right|^{2} \\
\leq & \frac{1}{\varepsilon}\left|u\left(x_{v}, r(v)\right)-u\left(\bar{y}_{(k-1) \Delta}+\frac{(v-k \Delta)}{\Delta}\left(\bar{y}_{k \Delta}-\bar{y}_{(k-1) \Delta}\right), r_{k}^{\Delta}\right)\right|^{2} \\
& +\frac{2}{1-\varepsilon}\left[T \int_{0}^{v}\left[f\left(x_{s}, r(s)\right)-f\left(\bar{y}_{s}, \bar{r}(s)\right)\right]^{2} d s+\left|\int_{0}^{v}\left[g\left(x_{s}, r(s)\right)-g\left(\bar{y}_{s}, \bar{r}(s)\right)\right] d w(s)\right|^{2}\right] .
\end{aligned}
$$

Assumption 2.4 yields

$$
\begin{aligned}
\mid u\left(x_{v},\right. & r(v))-\left.u\left(\bar{y}_{(k-1) \Delta}+\frac{v-k \Delta}{\Delta}\left(\bar{y}_{k \Delta}-\bar{y}_{(k-1) \Delta}\right), r_{k}^{\Delta}\right)\right|^{2} \\
\leq & 2 \kappa^{2}|| x_{v}-\bar{y}_{(k-1) \Delta}-\frac{v-k \Delta}{\Delta}\left(\bar{y}_{k \Delta}-\bar{y}_{(k-1) \Delta}\right) \|^{2} \\
& +2 \mid u\left(\bar{y}_{(k-1) \Delta}+\frac{v-k \Delta}{\Delta}\left(\bar{y}_{k \Delta}-\bar{y}_{(k-1) \Delta}\right), r(v)\right) \\
& -\left.u\left(\bar{y}_{(k-1) \Delta}+\frac{v-k \Delta}{\Delta}\left(\bar{y}_{k \Delta}-\bar{y}_{(k-1) \Delta}\right), r_{k}^{\Delta}\right)\right|^{2} \\
\leq & 2 \kappa^{2}||\left|x_{v}-y_{v}\right|+\left|y_{v}-\bar{y}_{v}\right|+\left|\bar{y}_{k \Delta}-\bar{y}_{(k-1) \Delta}\right|-\frac{v-k \Delta}{\Delta}\left|\bar{y}_{k \Delta}-\bar{y}_{(k-1) \Delta}\right| \|^{2} \\
& +2 \mid u\left(\bar{y}_{(k-1) \Delta}+\frac{v-k \Delta}{\Delta}\left(\bar{y}_{k \Delta}-\bar{y}_{(k-1) \Delta}\right), r(v)\right) \\
& -\left.u\left(\bar{y}_{(k-1) \Delta}+\frac{v-k \Delta}{\Delta}\left(\bar{y}_{k \Delta}-\bar{y}_{(k-1) \Delta}\right), r_{k}^{\Delta}\right)\right|^{2} \\
\leq & \frac{2 \kappa^{2}}{\varepsilon}\left\|x_{v}-y_{v}\right\|^{2}+\frac{4 \kappa^{2}}{1-\varepsilon}\left(\left\|y_{v}-\bar{y}_{v}\right\|^{2}+\left\|\bar{y}_{k \Delta}-\bar{y}_{(k-1) \Delta}\right\|^{2}\right) \\
& +2 \mid u\left(\bar{y}_{(k-1) \Delta}+\frac{v-k \Delta}{\Delta}\left(\bar{y}_{k \Delta}-\bar{y}_{(k-1) \Delta}\right), r(v)\right) \\
& -\left.u\left(\bar{y}_{(k-1) \Delta}+\frac{v-k \Delta}{\Delta}\left(\bar{y}_{k \Delta}-\bar{y}_{(k-1) \Delta}\right), r_{k}^{\Delta}\right)\right|^{2}
\end{aligned}
$$


Then, we have

$$
\begin{aligned}
|e(v)|^{2} \leq & \frac{2 \kappa^{2}}{\varepsilon^{2}}\left\|x_{v}-y_{v}\right\|^{2}+\frac{4 \kappa^{2}}{\varepsilon(1-\varepsilon)}\left(\left\|y_{v}-\bar{y}_{v}\right\|^{2}+\left\|\bar{y}_{k \Delta}-\bar{y}_{(k-1) \Delta}\right\|^{2}\right) \\
+ & +2 \mid u\left(\bar{y}_{(k-1) \Delta}+\frac{v-k \Delta}{\Delta}\left(\bar{y}_{k \Delta}-\bar{y}_{(k-1) \Delta}\right), r(v)\right) \\
& -\left.u\left(\bar{y}_{(k-1) \Delta}+\frac{v-k \Delta}{\Delta}\left(\bar{y}_{k \Delta}-\bar{y}_{(k-1) \Delta}\right), r_{k}^{\Delta}\right)\right|^{2} \\
& +\frac{2}{1-\varepsilon}\left[T \int_{0}^{v}\left[f\left(x_{s}, r(s)\right)-f\left(\bar{y}_{s}, \bar{r}(s)\right)\right]^{2} d s+\left|\int_{0}^{v}\left[g\left(x_{s}, r(s)\right)-g\left(\bar{y}_{s}, \bar{r}(s)\right)\right] d w(s)\right|^{2}\right] .
\end{aligned}
$$

Hence, for any $t_{1} \in[0, T]$, by Lemmas 3.2-3.5,

$$
\begin{aligned}
\mathbb{E}\left[\sup _{0 \leq t \leq t_{1}}\left|e\left(t \wedge \rho_{j}\right)\right|^{2}\right] \leq & \frac{2 \kappa^{2}}{\varepsilon^{2}} \mathbb{E}\left(\sup _{0 \leq t \leq t_{1}}\left\|x_{t \wedge \rho_{j}}-y_{t \wedge \rho_{j}}\right\|^{2}\right) \\
& +\frac{4 \kappa^{2}}{\varepsilon(1-\varepsilon)}\left[\mathbb{E}\left(\sup _{0 \leq t \leq t_{1}}\left\|y_{t \wedge \rho_{j}}-\bar{y}_{t \wedge \rho_{j}}\right\|^{2}\right)\right. \\
& \left.+\mathbb{E}\left(\sup _{0 \leq k \leq M-1}\left\|\bar{y}_{k \Delta \wedge \rho_{j}}-\bar{y}_{(k-1) \Delta \wedge \rho_{j}}\right\|^{2}\right)\right] \\
& +2 \mathbb{E}\left[\sup _{0 \leq k \leq M-1} \mid u\left(\bar{y}_{(k-1) \Delta}+\frac{k \Delta \wedge \rho_{j}-k \Delta}{\Delta}\left(\bar{y}_{k \Delta}-\bar{y}_{(k-1) \Delta}\right), r\left(k \Delta \wedge \rho_{j}\right)\right)\right. \\
& +\frac{2 T}{1-\varepsilon} \mathbb{E} \int_{0}^{t_{1} \wedge \rho_{j}}\left[f\left(x_{s}, r(s)\right)-f\left(\bar{y}_{s}\right), \bar{r}(s)\right]^{2} d s \\
& \left.+\left.\frac{2}{1-\varepsilon} \mathbb{E}\left[\overline{s u p}_{(k-1) \Delta}+\left.\frac{k \Delta \wedge \rho_{j}-k \Delta}{\Delta}\left(\int_{0 \leq t \leq t_{1}} \mid \int_{0}^{t \wedge \rho_{j}}\left[g\left(x_{s}, r(s)\right)-g\left(\bar{y}_{s}, \bar{r}_{(k-1) \Delta}\right), \bar{r}(k)\right)\right] d w(s)\right|^{2}\right]\right|^{2}\right] \\
& +\frac{2 \kappa^{2}}{\varepsilon^{2}} \mathbb{E}\left(\sup _{0 \leq t \leq t_{1}}\left\|x_{t \wedge \rho_{j}}-y_{t \wedge \rho_{j}}\right\|^{2}\right)+\frac{4 \kappa^{2}}{\varepsilon(1-\varepsilon)}(r(\Delta)+\beta(\Delta))+2 \zeta \Delta \\
& +\frac{2 T}{1-\varepsilon} \mathbb{E} \int_{0}^{t_{1} \wedge \rho_{j}}\left[f\left(x_{s}, r(s)\right)-f\left(\bar{y}_{s}, \bar{r}(s)\right)\right]^{2} d s \\
& \left.\sup _{0 \leq t \leq t_{1}}\left|\int_{0}^{t \wedge \rho_{j}}\left[g\left(x_{s}, r(s)\right)-g\left(\bar{y}_{s}, \bar{r}(s)\right)\right] d w(s)\right|^{2}\right] .
\end{aligned}
$$


Since $x(t)=y(t)=\xi(t)$ when $t \in[-\tau, 0]$, we have

$$
\begin{aligned}
\mathbb{E}\left(\sup _{0 \leq t \leq t_{1}}\left\|x_{t \wedge \rho_{j}}-y_{t \wedge \rho_{j}}\right\|^{2}\right) & \leq \mathbb{E}\left(\sup _{-\tau \leq \theta \leq 0} \sup _{0 \leq t \leq t_{1}}\left|x\left(t \wedge \rho_{j}+\theta\right)-y\left(t \wedge \rho_{j}+\theta\right)\right|^{2}\right) \\
& \leq \mathbb{E}\left(\sup _{-\tau \leq t \leq t_{1}}\left|x\left(t \wedge \rho_{j}\right)-y\left(t \wedge \rho_{j}\right)\right|^{2}\right) \\
& =\mathbb{E}\left(\sup _{0 \leq t \leq t_{1}}\left|x\left(t \wedge \rho_{j}\right)-y\left(t \wedge \rho_{j}\right)\right|^{2}\right) .
\end{aligned}
$$

By Assumption 2.2, Lemma 3.3, and Lemma 3.6, we may compute

$$
\begin{aligned}
\mathbb{E} \int_{0}^{t_{1} \wedge \rho_{j}} & \left|f\left(x_{s}, r(s)\right)-f\left(\bar{y}_{s}, \bar{r}(s)\right)\right|^{2} d s \\
\leq & \mathbb{E} \int_{0}^{t_{1} \wedge \rho_{j}}\left|f\left(x_{s}, r(s)\right)-f\left(\bar{y}_{s}, r(s)\right)+f\left(\bar{y}_{s}, r(s)\right)-f\left(\bar{y}_{s}, \bar{r}(s)\right)\right|^{2} d s \\
\leq & 2 C_{j} \mathbb{E} \int_{0}^{t_{1} \wedge \rho_{j}}\left\|x_{s}-y_{s}+y_{s}-\bar{y}_{s}\right\|^{2} d s+2 C \Delta \\
\leq & 4 C_{j} \mathbb{E} \int_{0}^{t_{1} \wedge \rho_{j}}\left\|x_{s}-y_{s}\right\|^{2} d s+4 C_{j} \mathbb{E} \int_{0}^{t_{1} \wedge \rho_{j}}\left\|y_{s}-\bar{y}_{s}\right\|^{2} d s+2 C \Delta \\
\leq & 4 C_{j} \mathbb{E} \int_{0}^{t_{1} \wedge \rho_{j}} \sup _{-\tau \leq \theta \leq 0}|x(s+\theta)-y(s+\theta)|^{2} d s+4 C_{j} T \beta(\Delta)+2 C \Delta \\
\leq & 4 C_{j} \mathbb{E} \int_{0}^{t_{1}} \sup _{-\tau \leq \theta \leq 0}\left|x\left(s \wedge \rho_{j}+\theta\right)-y\left(s \wedge \rho_{j}+\theta\right)\right|^{2} d s \\
& +4 C_{j} T \beta(\Delta)+2 C \Delta \\
\leq & 4 C_{j} \mathbb{E} \int_{0}^{t_{1}} \sup _{-\tau \leq r \leq s}\left|x\left(r \wedge \rho_{j}\right)-y\left(r \wedge \rho_{j}\right)\right|^{2} d s+4 C_{j} T \beta(\Delta)+2 C \Delta \\
= & 4 C_{j} \int_{0}^{t_{1}} \mathbb{E} \sup _{0 \leq r \leq s}\left|x\left(r \wedge \rho_{j}\right)-y\left(r \wedge \rho_{j}\right)\right|^{2} d s+4 C_{j} T \beta(\Delta)+2 C \Delta .
\end{aligned}
$$

By the Doob martingale inequality, Lemma 3.3, Lemma 3.6, and Assumption 2.2, we compute

$$
\begin{aligned}
\mathbb{E}\left[\sup _{0 \leq t \leq t_{1}}\left|\int_{0}^{t \wedge \rho_{j}}\left[g\left(x_{s}, r(s)\right)-g\left(\bar{y}_{s}, \bar{r}(s)\right)\right] d w(s)\right|^{2}\right] \\
\quad=\mathbb{E}\left[\sup _{0 \leq t \leq t_{1}}\left|\int_{0}^{t \wedge \rho_{j}}\left[g\left(x_{s}, r(s)\right)-g\left(\bar{y}_{s^{\prime}} r(s)\right)+g\left(\bar{y}_{s^{\prime}} r(s)\right)-g\left(\bar{y}_{s}, \bar{r}(s)\right)\right] d w(s)\right|^{2}\right]
\end{aligned}
$$




$$
\begin{aligned}
& \leq 8 C_{j} \mathbb{E} \int_{0}^{t_{1} \wedge \rho_{j}}\left\|x_{s}-\bar{y}_{s}\right\|^{2} d s+8 C \Delta \\
& \leq 16 C_{j} \int_{0}^{t_{1}} \mathbb{E}\left(\sup _{0 \leq r \leq s}\left|x\left(r \wedge \rho_{j}\right)-y\left(r \wedge \rho_{j}\right)\right|^{2}\right) d s+16 C_{j} T \beta(\Delta)+8 C \Delta .
\end{aligned}
$$

Therefore, (4.17) can be written as

$$
\begin{aligned}
\left(1-\frac{2 \kappa^{2}}{\varepsilon^{2}}\right) \mathbb{E}\left[\sup _{0 \leq t \leq t_{1}}\left|e\left(t \wedge \rho_{j}\right)\right|^{2}\right] \leq & \frac{4 \kappa^{2}}{\varepsilon(1-\varepsilon)}[\beta(\Delta)+\gamma(\Delta)]+\frac{8 C_{j} T(T+4)}{1-\varepsilon} \beta(\Delta)+\frac{4 C \Delta(T+8)}{1-\varepsilon} \\
& +2 \zeta \Delta+\frac{8 C_{j}(T+4)}{1-\varepsilon} \int_{0}^{t_{1}} \mathbb{E}\left(\sup _{0 \leq v \leq s}\left|e\left(v \wedge \rho_{j}\right)\right|^{2}\right) d s .
\end{aligned}
$$

Choosing $\varepsilon=(1+\sqrt{2} \kappa) / 2$ and noting $\kappa \in(0,1)$, we have

$$
\begin{aligned}
& \mathbb{E}\left(\sup _{0 \leq t \leq t_{1}}\left|e\left(t \wedge \rho_{j}\right)\right|^{2}\right) \\
& \leq \frac{16 \kappa^{2}(1+\sqrt{2} \kappa)}{(1-\sqrt{2} \kappa)^{2}(1+3 \sqrt{2} \kappa)}[\beta(\Delta)+\gamma(\Delta)]+\frac{16 C_{j} T(1+\sqrt{2} \kappa)^{2}(T+4)}{(1-\sqrt{2} \kappa)^{2}(1+3 \sqrt{2} \kappa)} \beta(\Delta) \\
& \quad+\frac{8 C \Delta(T+8)(1+\sqrt{2} \kappa)^{2}}{(1-\sqrt{2} \kappa)^{2}(1+3 \sqrt{2} \kappa)}+\frac{2(1+\sqrt{2} \kappa)^{2}}{(1-\sqrt{2} \kappa)^{2}(1+3 \sqrt{2} \kappa)} \zeta \Delta \\
&+\frac{16 C_{j}(1+\sqrt{2} \kappa)^{2}(T+4)}{(1-\sqrt{2} \kappa)^{2}(1+3 \sqrt{2} \kappa)} \int_{0}^{t_{1}} \mathbb{E}\left(\sup _{0 \leq s \leq v}\left|e\left(s \wedge \rho_{j}\right)\right|^{2}\right) d v \\
& \leq \frac{16}{(1-\sqrt{2} \kappa)^{2}}[\beta(\Delta)+\gamma(\Delta)]+\frac{16 C_{j} T(T+4)}{(1-\sqrt{2} \kappa)^{2}} \beta(\Delta)+\frac{8 C \Delta(T+8)}{(1-\sqrt{2} \kappa)^{2}} \\
&+\frac{2}{(1-\sqrt{2} \kappa)} \zeta \Delta+\frac{16 C_{j}(T+4)}{(1-\sqrt{2} \kappa)^{2}} \int_{0}^{t_{1}} \mathbb{E}\left(\sup _{0 \leq s \leq v}\left|e\left(s \wedge \rho_{j}\right)\right|^{2}\right) d v
\end{aligned}
$$


By the Gronwall inequality, we have

$$
\begin{aligned}
\mathbb{E}\left[\sup _{0 \leq t \leq t_{1}}\left|e\left(t \wedge \rho_{j}\right)\right|^{2}\right] \leq & {\left[\frac{16}{(1-\sqrt{2} \kappa)^{2}}(\beta(\Delta)+\gamma(\Delta))+\frac{16 C_{j} T(T+4)}{(1-\sqrt{2} \kappa)^{2}} \beta(\Delta)\right.} \\
& \left.+\frac{8 C \Delta(T+8)}{(1-\sqrt{2} \kappa)^{2}}+\frac{2 \zeta \Delta}{(1-\sqrt{2} \kappa)}\right] \times e^{\left(16 /(1-\sqrt{2} \kappa)^{2}\right) C_{j} T(T+4)}
\end{aligned}
$$

By (4.13),

$$
\begin{aligned}
\mathbb{E}\left(\sup _{0 \leq t \leq T}|e(t)|^{2}\right) \leq & {\left[\frac{16}{(1-\sqrt{2} \kappa)^{2}}(\beta(\Delta)+\gamma(\Delta))+\frac{16 C_{j} T(T+4)}{(1-\sqrt{2} \kappa)^{2}} \beta(\Delta)+\frac{8 C \Delta(T+8)}{(1-\sqrt{2} \kappa)^{2}}\right.} \\
& \left.+\frac{2 \zeta \Delta}{(1-\sqrt{2} \kappa)}\right] \times e^{\left(16 /(1-\sqrt{2} \kappa)^{2}\right) C_{j} T(T+4)}+\frac{2^{p+1} \delta \widetilde{H}}{p}+\frac{2(p-2) \widetilde{H}}{p \delta^{2 /(p-2)} j^{p}} .
\end{aligned}
$$

Given any $\epsilon>0$, we can now choose $\delta$ sufficient small such that $2^{p+1} \delta \widetilde{H} / p \leq \epsilon / 3$, then choose $j$ sufficient large such that

$$
\frac{2(p-2) \widetilde{H}}{p \delta^{2 /(p-2)} j^{p}}<\frac{\epsilon}{3}
$$

and finally choose $\Delta$ so small such that

$$
\begin{aligned}
& {\left[\frac{16}{(1-\sqrt{2} \kappa)^{2}}(\beta(\Delta)+\gamma(\Delta))+\frac{16 C_{j} T(T+4)}{(1-\sqrt{2} \kappa)^{2}} \beta(\Delta)+\frac{8 C \Delta(T+8)}{(1-\sqrt{2} \kappa)^{2}}+\frac{2 \zeta \Delta}{(1-\sqrt{2} \kappa)}\right]} \\
& \quad \times e^{\left(16 /(1-\sqrt{2} \kappa)^{2}\right) C_{j} T(T+4)}<\frac{\epsilon}{3}
\end{aligned}
$$

and thus $\mathbb{E}\left(\sup _{0 \leq t \leq T}|e(t)|^{2}\right) \leq \epsilon$ as required.

Remark 4.1. Obviously, according to Theorem 2.7, for neutral stochastic delay differential equations with Markovian switching [19], we can easily obtain that the numerical solutions converge to the true solutions in mean square under Assumptions 2.2-2.4.

\section{Convergence Order of the EM Method}

To reveal the convergence order of the EM method, we need the following assumptions. 
Assumption 5.1. There exists a constant $Q$ such that for all $\varphi, \psi \in C\left([-\tau, 0] ; \mathbb{R}^{n}\right), i \in S$, and $t \in[0, T]$,

$$
|f(\varphi, i)-f(\psi, i)|^{2} \vee|g(\varphi, i)-g(\psi, i)|^{2} \leq Q\|\varphi-\psi\|^{2}
$$

It is easy to see from the global Lipschitz condition that, for any $\varphi \in C\left([-\tau, 0] ; \mathbb{R}^{n}\right)$,

$$
|f(\varphi, i)|^{2} \vee|g(\psi, i)|^{2} \leq 2\left(|f(0, i)|^{2} \vee|g(0, i)|^{2}\right)+Q\|\varphi\|^{2}
$$

In other words, the global Lipschitz condition implies linear growth condition with the growth coefficient

$$
K=2\left[|f(0, i)|^{2} \vee|g(0, i)|^{2} \vee Q\right]
$$

Assumption 5.2. $\xi \in L_{\mathcal{F}_{0}}^{p}\left([-\tau, 0] ; \mathbb{R}^{n}\right)$ for some $p \geq 2$, and there exists a positive constant $\lambda$ such that

$$
\mathbb{E}\left(\sup _{-\tau \leq s \leq t \leq 0}|\xi(t)-\xi(s)|^{2}\right) \leq \lambda(t-s) .
$$

We can state another theorem, which reveals the order of the convergence.

Theorem 5.3. If Assumptions 5.1, 5.2, and 2.4 hold, for any positive constant $l>1$,

$$
\mathbb{E}\left(\sup _{0 \leq t \leq T}|x(t)-y(t)|^{2}\right) \leq O\left(\Delta^{1-1 / l}\right) .
$$

Proof. Since $\alpha(\Delta)$ may be replaced by $\lambda \Delta$, from Lemmas 3.2 and 3.3, there exist constants $\widetilde{c}_{1}(l)$ and $\widetilde{c}_{2}(l)$ such that $\beta(\Delta) \leq \widetilde{c}_{1}(l) \Delta^{(l-1) / l}$ and $\gamma(\Delta) \leq \widetilde{c}_{2}(l) \Delta^{(l-1) / l}$. Here we do not need to define the stopping times $u_{j}$ and $v_{j}$, and we may repeat the proof in Section 4 and directly compute

$$
\begin{aligned}
\mathbb{E}\left(\sup _{0 \leq t \leq T}|e(t)|^{2}\right) \leq & {\left[\frac{16}{(1-\sqrt{2} \kappa)^{2}}\left(\widetilde{c}_{1}(l)+\widetilde{c}_{2}(l)\right)+\frac{16 Q T(T+4)}{(1-\sqrt{2} \kappa)^{2}} \widetilde{c}_{1}(l)+\frac{8 C(T+8)}{(1-\sqrt{2} \kappa)^{2}}+\frac{2 \zeta}{(1-\sqrt{2} \kappa)}\right] } \\
& \times e^{\left(16 /(1-\sqrt{2} \kappa)^{2}\right) Q T(T+4)} \Delta^{(l-1) / l} \\
\leq & O\left(\Delta^{(l-1) / l}\right) .
\end{aligned}
$$

The proof is complete. 


\section{Conclusion}

The EM method for neutral stochastic functional differential equations with Markovian switching is studied. The results show that the numerical solution converges to the true solution under the local Lipschitz condition. In addition, the results also show that the order of convergence of the numerical method is close to 1 , although the order of the strong convergence in mean square for the EM scheme applied to both SDEs and SFDEs is one $[6,7,11]$ under the global Lipschitz condition. Hence, we can control the numerical solution's error; this method may value some path-dependent options more quickly and simply [25].

\section{Acknowledgments}

the work was supported by the Fundamental Research Funds for the Central Universities under Grant 2012089, China Postdoctoral Science Foundation funded project under Grant 2012M511615, the Research Fund for Wuhan Polytechnic University, and the State Key Program of National Natural Science of China (Grant no. 61134012).

\section{References}

[1] M. Mariton, Jump Linear Systems in Automatic Control, Marcel Dekker, New York, NY, USA, 1990.

[2] Y. Liu, "Numerical solution of implicit neutral functional-differential equations," SIAM Journal on Numerical Analysis, vol. 36, no. 2, pp. 516-528, 1999.

[3] D. Bahuguna and S. Agarwal, "Approximations of solutions to neutral functional differential equations with nonlocal history conditions," Journal of Mathematical Analysis and Applications, vol. 317, no. 2, pp. 583-602, 2006.

[4] C. T. H. Baker and E. Buckwar, "Numerical analysis of explicit one-step methods for stochastic delay differential equations," LMS Journal of Computation and Mathematics, vol. 3, pp. 315-335, 2000.

[5] X. Mao, "Numerical solutions of stochastic functional differential equations," LMS Journal of Computation and Mathematics, vol. 6, pp. 141-161, 2003.

[6] X. Mao, Stochastic Differential Equations and Applications, Horwood, Chichester, UK, 2nd edition, 2008.

[7] P. E. Kloeden and E. Platen, Numerical Solution of Stochastic Differential Equations, vol. 23, Springer, Berlin, Germany, 1992.

[8] Y. Shen, Q. Luo, and X. Mao, "The improved LaSalle-type theorems for stochastic functional differential equations," Journal of Mathematical Analysis and Applications, vol. 318, no. 1, pp. 134-154, 2006.

[9] X. Mao and S. Sabanis, "Numerical solutions of stochastic differential delay equations under local Lipschitz condition," Journal of Computational and Applied Mathematics, vol. 151, no. 1, pp. 215-227, 2003.

[10] C. Yuan and X. Mao, "Convergence of the Euler-Maruyama method for stochastic differential equations with Markovian switching," Mathematics and Computers in Simulation, vol. 64, no. 2, pp. 223-235, 2004.

[11] X. Mao and C. Yuan, Stochastic Differential Equations with Markovian Switching, Imperial College Press, London, UK, 2006.

[12] X. Mao, "Stochastic functional differential equations with Markovian switching," Functional Differential Equations, vol. 6, no. 3-4, pp. 375-396, 1999.

[13] X. Mao, C. Yuan, and G. Yin, "Approximations of Euler-Maruyama type for stochastic differential equations with Markovian switching, under non-Lipschitz conditions," Journal of Computational and Applied Mathematics, vol. 205, no. 2, pp. 936-948, 2007.

[14] D. J. Higham, X. Mao, and C. Yuan, "Preserving exponential mean-square stability in the simulation of hybrid stochastic differential equations," Numerische Mathematik, vol. 108, no. 2, pp. 295-325, 2007.

[15] F. Jiang, Y. Shen, and L. Liu, "Taylor approximation of the solutions of stochastic differential delay equations with Poisson jump," Communications in Nonlinear Science and Numerical Simulation, vol. 16, no. 2, pp. 798-804, 2011. 
[16] C. Yuan and W. Glover, "Approximate solutions of stochastic differential delay equations with Markovian switching," Journal of Computational and Applied Mathematics, vol. 194, no. 2, pp. 207-226, 2006.

[17] S. Zhu, Y. Shen, and L. Liu, "Exponential stability of uncertain stochastic neural networks wiht Markovian switching," Neural Processing Letters, vol. 32, pp. 293-309, 2010.

[18] L. Liu, Y. Shen, and F. Jiang, "The almost sure asymptotic stability and $p$ th moment asymptotic stability of nonlinear stochastic differential systems with polynomial growth," IEEE Transactions on Automatic Control, vol. 56, no. 8, pp. 1985-1990, 2011.

[19] S. Zhou and F. Wu, "Convergence of numerical solutions to neutral stochastic delay differential equations with Markovian switching," Journal of Computational and Applied Mathematics, vol. 229, no. 1, pp. 85-96, 2009.

[20] F. Wu and X. Mao, "Numerical solutions of neutral stochastic functional differential equations," SIAM Journal on Numerical Analysis, vol. 46, no. 4, pp. 1821-1841, 2008.

[21] A. Bellen, Z. Jackiewicz, and M. Zennaro, "Stability analysis of one-step methods for neutral delaydifferential equations," Numerische Mathematik, vol. 52, no. 6, pp. 605-619, 1988.

[22] F. Hartung, T. L. Herdman, and J. Turi, “On existence, uniqueness and numerical approximation for neutral equations with state-dependent delays," Applied Numerical Mathematics, vol. 24, no. 2-3, pp. 393-409, 1997.

[23] Z. Jackiewicz, "One-step methods of any order for neutral functional differential equations," SIAM Journal on Numerical Analysis, vol. 21, no. 3, pp. 486-511, 1984.

[24] K. Liu and X. Xia, "On the exponential stability in mean square of neutral stochastic functional differential equations," Systems $\mathcal{E}$ Control Letters, vol. 37, no. 4, pp. 207-215, 1999.

[25] F. Jiang, Y. Shen, and F. Wu, "Convergence of numerical approximation for jump models involving delay and mean-reverting square root process," Stochastic Analysis and Applications, vol. 29, no. 2, pp. 216-236, 2011. 


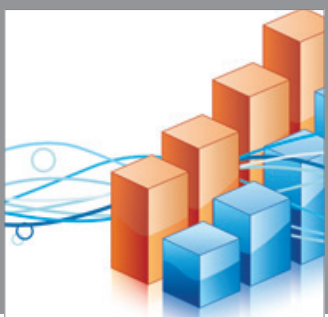

Advances in

Operations Research

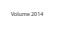

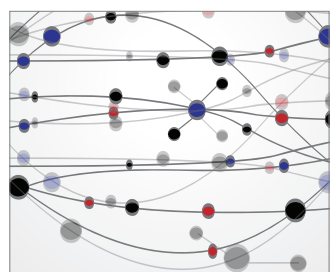

\section{The Scientific} World Journal
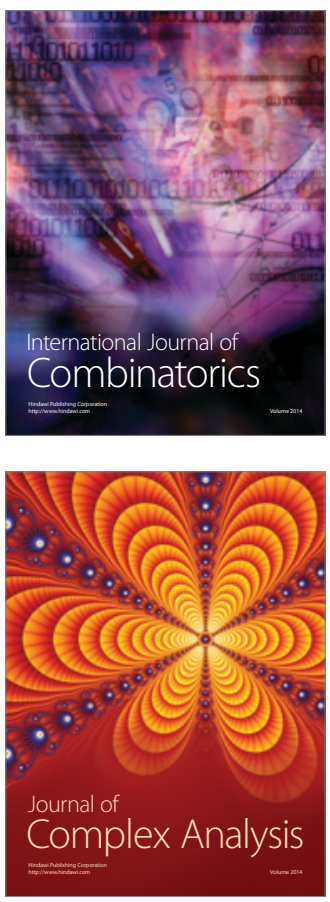

International Journal of

Mathematics and

Mathematical

Sciences
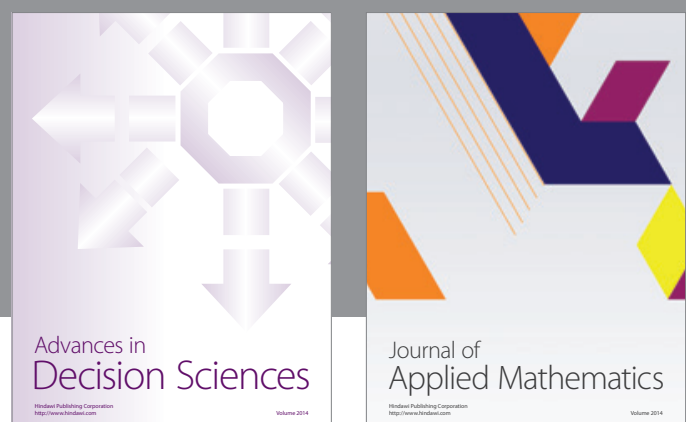

Journal of

Applied Mathematics
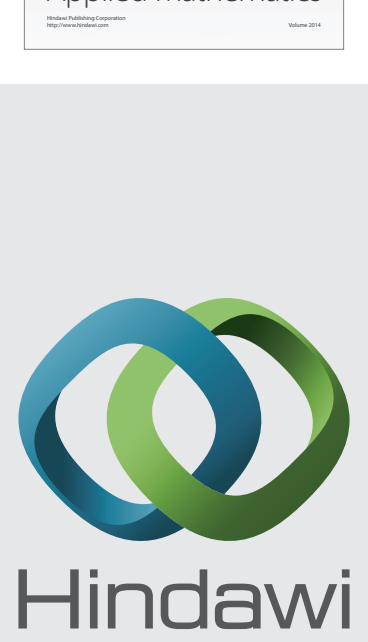

Submit your manuscripts at http://www.hindawi.com
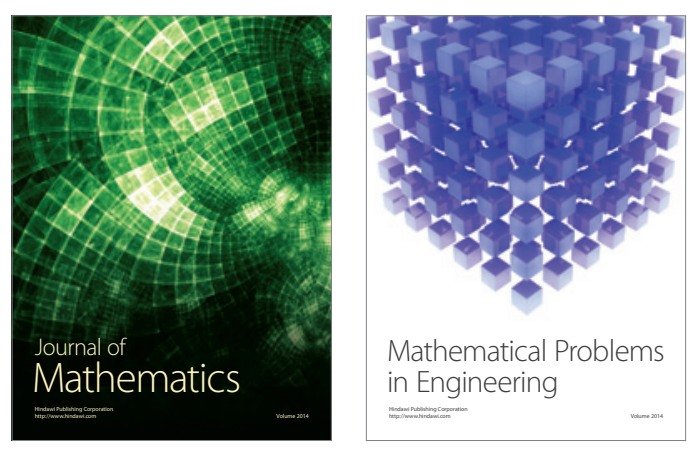

Mathematical Problems in Engineering
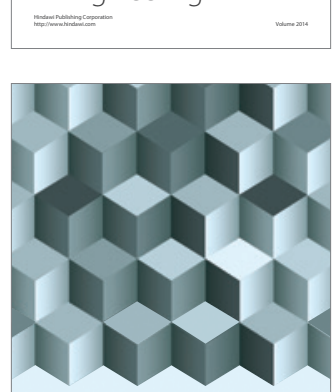

Journal of

Function Spaces
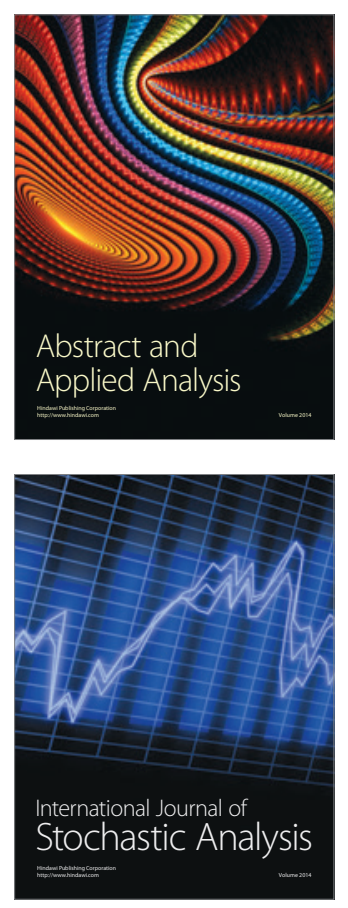

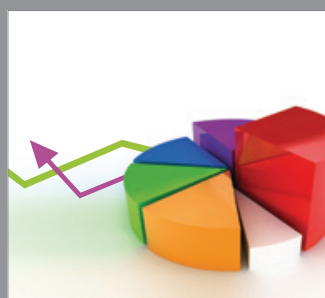

ournal of

Probability and Statistics

Promensencen
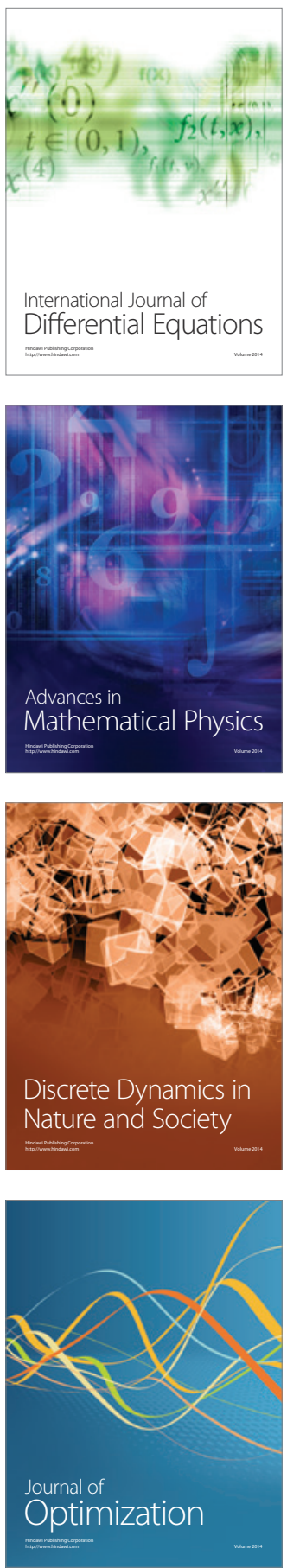